\title{
TILTING EQUIVALENCE FOR SUPERCONFORMAL ALGEBRAS
}

\author{
KENJI IOHARA and YOSHIYUKI KOGA
}

\section{Introduction}

In 1984, B. Feigin [5] observed a duality between Verma modules over the Virasoro algebra in terms of the non-vanishing condition of the semi-infinite torsion. The crucial number arising there is the critical dimension of the bosonic string theory. This phenomenon remained mysterious until the appearance of the work [1] by S. Arkhipov in 1997. There, he generalized this mysterious duality and explained it as an equivalence between certain categories of modules over a Z-graded associative algebra. The techniques used there are some deep results from semi-infinite homological algebra (e.g., see [14]) which seems to be still complicated, at least for the authors. Soon after, in 1998, W. Soergel [12] found a key to explain this duality for a Z-graded Lie algebra, and as an application he determined characters of tilting modules over symmetrizable Kac-Moody algebras. W. Soergel's technique is applicable to a Z-graded Lie superlalgebra. Indeed, J. Brundan [2] imitated Soergel's construction and computed characters of tilting modules over some finite dimensional Lie superalgebras. Here, we should notice that W. Soergel and J. Brundan both assumed that a Z-graded Lie superalgebra is generated by its local part, i.e., $\mathfrak{g}^{1} \oplus \mathfrak{g}^{0} \oplus \mathfrak{g}^{-1}$ to simplify some arguments.

Several important superconformal algebras [9], even the Virasoro algebra, do not satisfy their assumption on Z-graded Lie (super)algebras. The purpose of this paper is to make such an equivalence applicable to (non-trivial central extensions of) superconformal algebras which seems to be significant in its application to physics such as superstring theory. Here we generalize arguments due to W. Soergel. Moreover, we describe the equivalence explicitly by computing semi-infinite characters (Definition 2.1) of central extensions of superconformal algebras. Here, we also compute semi-infinite characters of contragredient Lie superalgebras.

This paper is organized as follows. In Section 2, we recall the definition of a semi-infinite character and state some properties. In Section 3, we construct

Received April 1, 2005. 
the semi-regular bimodule. In Section 4, using the semi-regular bimodule, we show categorical equivalences. In Section 5, we compute semi-infinite characters of superconformal algebras and contragradient Lie superalgebras.

Two authors are partially supported by JSPS Grant in Aid of Scientific Research. Part of this work was done when K. Iohara had been visiting KIAS, Korea Institute for Advanced Study. He would like to thank the members of the institute for their support and their warm hospitality.

\section{Critical cocycle and semi-infinite character}

In [12] and [2], 1-cocycle called semi-infinite character plays an essential role to construct the categorical equivalence. Here, in order to extend their arguments, we introduce semi-infinite characters for Z-graded Lie superalgebras not necessarily generated by its local part by means of the critical cocycle. Note that the results in Section 2.1-2.3 are straightforward generalizations of statements in [12] to the Lie superalgebra setting. Some results in Section 2.4 are new, although they might be known among specialists.

\subsection{Notation}

Throughout this paper, let $\mathrm{K}$ be a field whose characteristic is zero. For a $\mathrm{Z}_{2} \times \mathrm{Z}$-graded K-vector space $M=\bigoplus_{(\sigma, i) \in \mathrm{Z}_{2} \times \mathrm{Z}} M_{\sigma}^{i}$, set $M^{i}:=M_{\overline{0}}^{i} \oplus M_{\overline{1}}^{i}$ and $M_{\sigma}:=\bigoplus_{i \in Z} M_{\sigma}^{i}$. For $x \in M_{\sigma}$, we denote the parity $\sigma$ of $x$ by $|x|$ We also set $M^{\geq n}:=\bigoplus_{i \geq n} M^{i}$ and $M^{\leq n}:=\bigoplus_{i \leq n} M^{i}$. For simplicity, we denote $M^{\geq 1}$, $M^{\leq-1}, M^{\geq 0}$ and $M^{\leq 0}$ by $M^{+}, M^{-}, M^{\geq}$and $M^{\leq}$respectively.

For $\mathrm{Z} \times \mathrm{Z}_{2}$-graded $\mathrm{K}$-vector spaces $M$ and $N$, we set

$$
\mathscr{H} \operatorname{om}_{\mathrm{K}}(M, N):=\bigoplus_{(\sigma, i) \in \mathrm{Z}_{2} \times \mathrm{Z}} \operatorname{Hom}_{\mathrm{K}}(M, N)_{\sigma}^{i},
$$

where $\operatorname{Hom}_{\mathcal{K}}(M, N)_{\tau}^{j}:=\left\{f \in \operatorname{Hom}_{\mathrm{K}}(M, N) \mid f\left(M_{\sigma}^{i}\right) \subset M_{\sigma+\tau}^{i+j}, \forall(\sigma, i) \in\right.$ $\left.\mathrm{Z}_{2} \times \mathrm{Z}\right\}$. In particular, $\mathscr{H} \operatorname{om}_{\mathrm{K}}(M, N)$ is naturally $\mathrm{Z}_{2} \times \mathrm{Z}$-graded.

Let $\mathrm{K}_{\overline{0}}^{0}$ be the one dimensional $Z_{2} \times Z$-graded $\mathrm{K}$-vector space such that

$$
\operatorname{dim}\left(\mathrm{K}_{\overline{0}}^{0}\right)_{\sigma}^{i}=\left\{\begin{array}{ll}
1 & (\sigma, i)=(\overline{0}, 0) \\
0 & (\sigma, i) \neq(\overline{0}, 0)
\end{array} .\right.
$$

For a $Z_{2} \times Z$-graded $\mathrm{K}$-vector space $M$, we set

$$
M^{\circledast}:=\mathscr{H}_{0} \mathrm{~m}_{\mathrm{K}}\left(M, \mathrm{~K}_{\overline{0}}^{0}\right), \quad M^{*}:=\operatorname{Hom}_{\mathrm{K}}\left(M, \mathrm{~K}_{\overline{0}}^{0}\right) .
$$

Throughout this paper, for each $(\sigma, n) \in \mathrm{Z}_{2} \times \mathbf{Z}$, we regard $\left(M_{\sigma}^{n}\right)^{*}$ as a subspace of $M^{\circledast}$ in the following way: Let $\pi_{\sigma}^{n}: M \rightarrow M_{\sigma}^{i}$ be the projection with respect 
to $M=\bigoplus_{(\sigma, i) \in \mathrm{Z}_{2} \times \mathrm{Z}} M_{\sigma}^{i}$. For $\phi \in\left(M_{\sigma}^{n}\right)^{*}$, we identify $\phi$ with $\phi \circ \pi_{\sigma}^{n}$ and regard $\phi \in M^{\circledast}$.

For $Z_{2} \times \mathbf{Z}$-graded $\mathrm{K}$ vector space $M$ such that $\operatorname{dim} M^{i}<\infty$ for any $i \in \mathbf{Z}$, we set

$$
\operatorname{ch} M:=\sum_{i \in \mathbf{Z}}\left(\operatorname{dim} M^{i}\right) q^{i} \in \mathrm{Z}\left[\left[q, q^{-1}\right]\right] .
$$

\subsection{Critical cocycle}

Let $\mathrm{g}$ be a Z-graded Lie superalgebra over a field $\mathrm{K}$, i.e.,

$$
\mathfrak{g}=\bigoplus_{(\sigma, i) \in \mathbf{Z}_{2} \times \mathbf{Z}} \mathfrak{g}_{\sigma}^{i}, \quad\left[\mathfrak{g}_{\sigma}^{i}, \mathfrak{g}_{\tau}^{j}\right] \subset \mathfrak{g}_{\sigma+\tau}^{i+j} \quad\left(\forall i, j \in \mathbf{Z}, \sigma, \tau \in \mathbf{Z}_{2}\right) .
$$

$g$ has the following triangular decomposition

$$
\mathfrak{g}=\mathfrak{g}^{-} \oplus \mathfrak{g}^{0} \oplus \mathfrak{g}^{+} .
$$

In the sequel, we denote $\mathfrak{g}^{0}, \mathfrak{g}^{\geq}$and $\mathfrak{g}^{-}$by $\mathfrak{h}, \mathfrak{b}$ and $\mathfrak{n}$ for simplicity.

Throughout Section 2 and Section 3, let $\mathrm{g}$ be a Z-graded Lie superalgebra which satisfies

$$
\operatorname{dim} \mathrm{g}^{i}<\infty \quad\left(\forall i \in \mathbf{Z}_{<0}\right) .
$$

Let $\pi^{-}: \mathfrak{g} \rightarrow \mathfrak{n}$ be the projection with respect to the triangular decomposition (2), and let $i^{-}: \mathfrak{n} \hookrightarrow \mathfrak{g}$ be the inclusion map. We set $\pi:=i^{-} \circ \pi^{-}: \mathfrak{g} \rightarrow \mathfrak{g}$. We define the map $\omega \in \operatorname{Hom}_{\mathrm{K}}\left(\mathfrak{g} \otimes_{\mathrm{K}} \mathfrak{g}, \mathrm{K}_{\overline{0}}^{0}\right)$ as follows:

$$
\omega(u, v):=\operatorname{str}_{\mathfrak{g}}([\pi \circ \operatorname{ad} u, \pi \circ \operatorname{ad} v]-\pi \circ[\operatorname{ad} u, \operatorname{ad} v]),
$$

where $\operatorname{str}_{\mathfrak{g}}$ denotes the supertrace on $\mathfrak{g}$.

By the following lemma and the assumption (3), $\omega$ is well-defined.

Lemma 2.1. Suppose that $u \in \mathrm{g}_{\sigma}^{i}$ and $v \in \mathfrak{g}_{\tau}^{j}$.

(1) If $(i, \sigma)+(j, \tau) \neq(0, \overline{0})$ or $i=j=0$, then $\omega(u, v)=0$.

(2) If $i=n, j=-n\left(n \in \mathbf{Z}_{>0}\right)$, then

$$
\omega(u, v)=(-1)^{|u|} \operatorname{str}_{\oplus_{m=1}^{n} \mathrm{~g}^{-m}}(\operatorname{ad} v \circ \operatorname{ad} u) .
$$

Moreover, one can directly check that $\omega$ satisfies the two cocycle conditions. The map $\omega$ is called the critical cocycle of $g$.

In the sequel, let

$$
\left\{x_{k}\right\}_{k \in I^{+}}, \quad\left\{h_{k}\right\}_{k \in I^{0}}, \quad\left\{y_{k}\right\}_{k \in I^{-}}
$$


be K-basis of $\mathfrak{g}^{+}, \mathfrak{g}^{0}$ and $\mathfrak{g}^{-}$, whose basis vectors consist of homogeneous vectors with respect to the $\mathrm{Z}_{2} \times \mathbf{Z}$-gradation of g. For $u, v \in \mathfrak{g}$, we define constants $C_{u, v}^{x_{k}}, C_{u, v}^{h_{k}}$ and $C_{u, v}^{y_{k}}$ by

$$
[u, v]=\sum_{k \in I^{+}} C_{u, v}^{x_{k}} x_{k}+\sum_{k \in I^{0}} C_{u, v}^{h_{k}} h_{k}+\sum_{k \in I^{-}} C_{u, v}^{y_{k}} y_{k} .
$$

Then, the following lemma holds.

Lemma 2.2. Suppose that $u \in \mathfrak{g}^{n}$ and $v \in \mathfrak{g}^{-n}\left(n \in \mathbf{Z}_{>0}\right)$. Then, we have

$$
\omega(u, v)=\sum_{k \in I^{0}} \sum_{m \in I^{-}}(-1)^{|u|+\left|h_{k}\right|} C_{y_{m}, u}^{h_{k}} C_{h_{k}, v}^{y_{m}}+\sum_{k \in I^{+}} \sum_{m \in I^{-}}(-1)^{|u|+\left|x_{k}\right|} C_{y_{m}, u}^{x_{k}} C_{x_{k}, v}^{y_{m}} .
$$

\subsection{Semi-infinite character}

To construct the semi-regular bimodule over $\mathfrak{g}$, we assume that

$$
\exists \eta \in \operatorname{Hom}_{\mathrm{K}}\left(\mathfrak{g}, \mathrm{K}_{\overline{0}}^{0}\right) \quad \text { such that } \omega=d \eta,
$$

where $d \eta \in \operatorname{Hom}_{\mathrm{K}}\left(\mathfrak{g} \wedge \mathfrak{g}, \mathrm{K}_{\overline{0}}^{0}\right)$ is defined by $d \eta(u, v):=-\eta([u, v])$.

For $\gamma \in\left(\mathfrak{h}_{\overline{0}}\right)^{*}$, we regard $\gamma \in \mathfrak{g}^{\circledast} \subset \operatorname{Hom}_{\mathfrak{K}}\left(\mathfrak{g}, \mathrm{K}_{\overline{0}}^{0}\right)$ as in Section 2.1.

Definition 2.1 (cf. [12]). Let $\omega$ be the critical cocycle of $\mathfrak{g} . \gamma \in\left(\mathfrak{h}_{\overline{0}}\right)^{*}$ is called a semi-infinite character of $\mathfrak{g}$ if $d \gamma=\omega$.

Remark that under the assumption (7), a semi-infinite character of $g$ exists, and if $\mathfrak{h}_{\overline{0}} \subset[\mathfrak{g}, \mathfrak{g}]$, then $\gamma$ is unique. Moreover, by Lemma 2.1, we have

$$
\gamma\left(\mathfrak{g}_{\sigma}^{n}\right)=\{0\}((\sigma, n) \neq(\overline{0}, 0)), \quad \gamma\left(\left[\mathfrak{h}_{\overline{0}}, \mathfrak{h}_{\overline{0}}\right]\right)=\{0\} .
$$

Hence, $\gamma$ is a character of $\mathfrak{h}_{\overline{0}}$.

The following two lemmata are useful to compute a semi-infinite character of a Z-graded Lie superalgebra explicitly.

Lemma 2.3. Let a be a Z-graded Lie superalgebra, which satisfies (3). (We do not assume (7).) Suppose that $\mathfrak{a}$ is generated by the subspace $\bigoplus_{|i| \leq n} \mathfrak{a}^{i}$ for some $n \in Z_{>0}$. Let $\omega$ be the critical cocycle of $\mathfrak{a}$. If there exists $\gamma \in\left(\mathfrak{a}^{0}\right)^{*}$ such that

$$
\omega(u, v)=-\gamma([u, v]) \quad\left(\forall u \in \mathfrak{a}^{i}, v \in \mathfrak{a}^{-i}\right)
$$

holds for any $i(0 \leq i \leq n)$, then $\gamma$ is a semi-infinite character of $a$.

Lemma 2.4. Let $\alpha: \mathfrak{a} \rightarrow \mathfrak{g}$ be a central extension of a Z-graded Lie superalgebra g. Suppose that $\operatorname{Ker} \alpha \subset \mathfrak{a}^{0}$. If $\gamma$ is a semi-infinite character of $\mathfrak{g}$, then $\gamma \circ \alpha$ is a semi-infinite character of $\mathfrak{a}$. 


\subsection{Remark on the BRST differential}

It is known that the critical cocycle of a Z-graded Lie algebra also appears in the vanishing condition of the square of the BRST differential (cf. [14]). Here, we explain a similar relation between a semi-infinite character and the BRST differentials in the super setting.

Only in this subsection, we further assume that a Z-graded Lie superalgebra g satisfies $\operatorname{dim} \mathrm{g}^{n}<\infty$ for all $n \in \mathrm{Z}$. Here, let $\left\{e_{i} \mid i \in \mathscr{I}\right\}$ be a K-basis of $\mathrm{g}$ with vectors which are homogeneous with respect to the $Z_{2} \times Z$-gradation of g. Set $\mathscr{I} \geq:=\left\{i \in \mathscr{I} \mid e_{i} \in \mathfrak{b}\right\}$ and $\mathscr{I}^{-}:=\left\{i \in \mathscr{I} \mid e_{i} \in \mathfrak{n}\right\}$. Let $\left\{e_{i}^{\prime} \mid i \in \mathscr{I}\right\}$ be the dual basis of $\left\{e_{i} \mid i \in \mathscr{I}\right\}$.

Let $\langle\rangle:, \mathrm{g}^{\circledast} \times \mathfrak{g} \rightarrow \mathrm{K}$ be the dual pairing defined by $\langle f, v\rangle:=f(v)$ for $f \in \mathfrak{g}^{\circledast}$ and $v \in \mathfrak{g}$. We extend $\langle$,$\rangle to the non-degenerate supersymmetric$ bilinear form on the space $\mathfrak{g} \oplus \mathfrak{g}^{\circledast}$ by

$$
\langle v, f\rangle=-(-1)^{(1-|v|)(1-|f|)}\langle f, v\rangle \quad\left(f \in \mathfrak{g}^{\circledast}, v \in \mathfrak{g}\right)
$$

and $\langle\mathfrak{g}, \mathfrak{g}\rangle=\{0\}=\left\langle\mathfrak{g}^{\circledast}, \mathfrak{g}^{\circledast}\right\rangle$.

We recall the definition of the Clifford superalgebra $C\left(\mathfrak{g} \oplus \mathfrak{g}^{\circledast}\right)$ on $\mathfrak{g} \oplus \mathfrak{g}^{\circledast}$. Let $T\left(\mathfrak{g} \oplus \mathfrak{g}^{\circledast}\right)$ be the tensor algebra on $\mathfrak{g} \oplus \mathfrak{g}^{\circledast}$, and let $J$ be the two-sided ideal of $T\left(\mathfrak{g} \oplus \mathfrak{g}^{\circledast}\right)$ generated by the following vectors:

$$
\left\{x \otimes y-(-1)^{(1-|x|)(1-|y|)} y \otimes x-\langle x, y\rangle \mid x, y \in \mathfrak{g} \oplus \mathfrak{g}^{\circledast}\right\} .
$$

Define $C\left(\mathfrak{g} \oplus \mathfrak{g}^{\circledast}\right):=T\left(\mathfrak{g} \oplus \mathfrak{g}^{\circledast}\right) / J$. Remark that with respect to the natural filtered structure on $C\left(\mathfrak{g} \oplus \mathrm{g}^{\circledast}\right)$, we have

$$
\operatorname{gr} C\left(\mathfrak{g} \oplus \mathfrak{g}^{\circledast}\right) \simeq S\left(\Pi g \oplus \Pi g^{\circledast}\right),
$$

where $\Pi$ denotes the parity shift operator and $S\left(\Pi g \oplus \Pi g^{\circledast}\right)$ is the symmetric algebra on $\Pi g \oplus \Pi g^{\circledast}$. Hence, there exists the canonical inclusion $\Pi g \oplus$ $\Pi \mathrm{g}^{\circledast} \hookrightarrow C\left(\mathfrak{g} \oplus \mathrm{g}^{\circledast}\right)$. Here, we denote the image $x \in \mathfrak{g} \oplus \mathfrak{g}^{\circledast}$ under the composition of the maps

$$
\mathfrak{g} \oplus \mathfrak{g}^{\circledast} \stackrel{\Pi}{\longrightarrow} \Pi \mathfrak{g} \oplus \Pi \mathfrak{g}^{\circledast} \hookrightarrow C\left(\mathfrak{g} \oplus \mathfrak{g}^{\circledast}\right)
$$

by $\bar{x}$.

Next, we introduce the space of semi-infinite forms $\bigwedge^{\frac{\infty}{2}+\bullet}[11]$. Let $C(\mathfrak{b} \oplus$ $\left.\mathfrak{n}^{\circledast}\right)$ be the subalgebra of $C\left(\mathfrak{g} \oplus \mathfrak{g}^{\circledast}\right)$ generated by $\mathfrak{b} \oplus \mathfrak{n}^{\circledast}$ and 1 , where we regard $\mathfrak{n}^{\circledast}=\bigoplus_{(\sigma, n) \in \mathrm{Z}_{2} \times \mathrm{Z}_{<0}}\left(\mathfrak{g}_{\sigma}^{n}\right)^{*} \subset \mathfrak{g}^{\circledast}$ as in Section 2.1 . Let $\mathrm{K} \mathbf{1}$ be the onedimensional representation of $C\left(\mathfrak{b} \oplus \mathfrak{n}^{\circledast}\right)$ defined by

$$
|\mathbf{1}|:=\overline{0}, \quad 1 . \mathbf{1}:=\mathbf{1}, \quad x . \mathbf{1}:=0 \quad\left(x \in \mathfrak{b} \oplus \mathfrak{n}^{\circledast}\right) .
$$


We set

$$
\bigwedge^{\frac{\infty}{2}+\bullet}:=C\left(\mathfrak{g} \oplus \mathfrak{g}^{\circledast}\right) \otimes_{C\left(\mathfrak{b} \oplus \mathfrak{n}^{\circledast)}\right.} \mathbf{K} \mathbf{1} .
$$

For each $\delta \in\left(\mathfrak{g}_{0}^{0}\right)^{*}\left(\subset \mathfrak{g}^{\circledast}\right)$, one can introduce projective $\mathfrak{g}$-module structure on $\bigwedge^{\frac{\infty}{2}+\bullet}$ as follow: For $x \in \mathfrak{g}$, we set $\eta_{\delta}(x) \in \operatorname{End}_{\mathrm{K}}\left(\Lambda^{\frac{\infty}{2}+\bullet}\right)$ by

$$
\left.\eta_{\delta}(x):=\delta(x)+\sum_{i \in \mathscr{I}}(-1)^{\left|e_{i}\right| \circ} \overline{{ }_{\circ}^{\prime}} \overline{\left[e_{i}, x\right]}\right]_{\circ}^{\circ},
$$

where the normal ordered product ${ }_{\circ}^{\circ} \cdot{ }_{\circ}^{\circ}$ is defined as follows: For $x \in \mathfrak{g}_{\sigma}^{m}$ and $y \in\left(g_{\tau}^{n}\right)^{*}$,

$$
\circ \bar{y} \bar{x}_{\circ}^{\circ}:=\left\{\begin{array}{ll}
\bar{y} \bar{x} & \text { if } m \geq 0 \\
(-1)^{(1-|x|)(1-|y|)} \bar{x} \bar{y} & \text { if } m<0
\end{array}\right. \text {. }
$$

By direct computation, we have

Proposition 2.1. For any $x, y \in \mathfrak{g}$, we have

$$
\left[\eta_{\delta}(x), \eta_{\delta}(y)\right]=\eta_{\delta}([x, y])+\mu_{\delta}(x, y),
$$

where $\mu_{\delta}$ is a 2-cocycle of g. The explicit form of $\mu_{\delta}$ is given by

$$
\mu_{\delta}:=d \delta+\sum_{i \in \mathscr{I} \geq} \sum_{j \in \mathscr{I}^{-}}(-1)^{\left|e_{j}\right|} \operatorname{ad}^{\prime} e_{j} \cdot e_{i}^{\prime} \wedge \operatorname{ad}^{\prime} e_{i} \cdot e_{j}^{\prime},
$$

where $d$ is the coboundary operator (Subsection 2.3) and

$$
\begin{aligned}
f \wedge g(u, v) & :=f(u) g(v)-(-1)^{|f||g|} g(u) f(v), \\
\left(\operatorname{ad}^{\prime} u \cdot f\right)(v) & :=-(-1)^{|u||f|} f(\operatorname{ad} u \cdot v)
\end{aligned}
$$

for $f, g \in \mathfrak{g}^{\circledast}$ and $u, v \in \mathfrak{g}$.

Moreover, by the formula (5), we have

LEMmA 2.5. For $u \in \mathrm{g}_{\sigma}^{n}$ and $v \in \mathfrak{g}_{\sigma}^{-n}\left(n \in \mathbf{Z}_{>0}\right)$,

$$
\sum_{i \in \mathscr{I} \geq} \sum_{j \in \mathscr{I}^{-}}(-1)^{\left|e_{j}\right|} \operatorname{ad}^{\prime} e_{j} \cdot e_{i}^{\prime} \wedge \operatorname{ad}^{\prime} e_{i} \cdot e_{j}^{\prime}(u, v)=\omega(u, v),
$$

where $\omega$ is the critical cocycle of $\mathrm{g}$.

Remark that, if $\delta=-\gamma$, where $\gamma$ is a semi-infinite character of $\mathfrak{g}$, then $\mu_{\delta}=0$ and $\eta_{\delta}$ defines $g$-module structure on $\bigwedge^{\frac{\infty}{2}+\bullet}$. 
Finally, we introduce the BRST differential and state its vanishing condition. For a Z-graded g-module $V=\bigoplus_{n \in Z} V^{n}$ which satisfies $V^{n}=\{0\}$ for $n \gg 0$, we define $Q \in \operatorname{End}_{\mathrm{K}}\left(V \otimes_{\mathrm{K}} \bigwedge^{\frac{\infty}{2}+\bullet}\right)$ by

$$
Q:=\operatorname{id}_{V} \otimes \bar{\delta}+\sum_{i \in \mathscr{I}} e_{i} \otimes \overline{e_{i}^{\prime}}-\frac{1}{2} \sum_{i, j \in \mathscr{I}}(-1)^{\left(\left|e_{i}\right|+\left|e_{j}\right|\right)\left|e_{j}\right|} \operatorname{id}_{V} \otimes_{\circ}^{\circ} \overline{\left[e_{i}, e_{j}\right]} \overline{e_{i}^{\prime}} \overline{e_{j \circ}^{\prime} \circ},
$$

where the normal ordered product ${ }_{\circ}^{\circ} \cdot{ }_{\circ}^{\circ}$ is defined as follows: For $x \in \mathfrak{g}_{\sigma}^{m}$ and $y_{i} \in\left(\mathfrak{g}_{\tau_{i}}^{n_{i}}\right)^{*}(i=1,2)$,

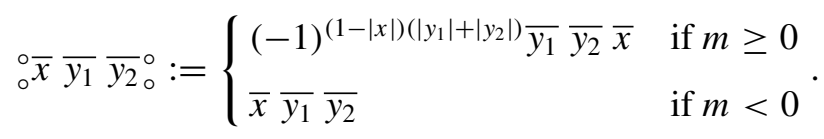

Proposition 2.2.

$$
Q^{2}=\mathrm{id}_{V} \otimes \overline{\mu_{\delta}}
$$

holds. In particular, if $\delta=-\gamma$, where $\gamma$ is a semi-infinite character of $\mathfrak{g}$, then $Q^{2}=0$.

\section{Semi-regular bimodule}

Using a semi-infinite character introduced in the previous section, we construct the semi-regular bimodule (or the semijective bimodule in [14]). In the case where a Lie superalgebra is generated by its local part, the semi-regular bimodule is constructed in [2].

\subsection{Preliminaries}

Let $\mathfrak{a}$ and $\mathfrak{b}$ be Z-graded Lie superalgebras. Let $M$ be a Z-graded $(\mathfrak{a}, \mathfrak{b})$ bimodule, and let $N$ be a $Z$-graded $\mathrm{K}$-vector space. In the sequel, except when we consider the antipode dual, we regard the space $\mathscr{H} \operatorname{om}_{\mathrm{K}}(M, N)$ as a Z-graded $(\mathfrak{b}, \mathfrak{a})$-bimodule via

(1) The left action:

$$
(b . \psi)(m):=(-1)^{|b|(|\psi|+|m|)} \psi(m . b),
$$

(2) The right action:

$$
(\psi \cdot a)(m):=\psi(a . m),
$$

for $a \in \mathfrak{a}, b \in \mathfrak{b}, \psi \in \mathscr{H} \mathrm{om}_{\mathfrak{K}}(M, N)$ and $m \in M$. Moreover, suppose that $\mathfrak{g}$ is a Z-graded subalgebra of $\mathfrak{a}$ and $N$ is a left $\mathfrak{g}$-module. Set

$$
\begin{aligned}
\mathscr{H}_{\mathfrak{5}}(M, N):=\left\{\psi \in \mathscr{H}_{\mathrm{K}}(M, N) \mid\right. & a .(\psi(m)) \\
& \left.=(-1)^{|a||\psi|} \psi(\text { a.m })(\forall a \in \mathfrak{S})\right\} .
\end{aligned}
$$


Then, it is a Z-graded left $\mathfrak{b}$-submodule of $\mathscr{H} \mathrm{om}_{\mathrm{K}}(M, N)$.

The following lemma is useful for later.

LeMmA 3.1. Let $\mathfrak{a}, \mathfrak{b}$ and $\mathfrak{c}$ be Z-graded Lie superalgebras, and L, M and $N$ be a $\mathrm{Z}$-graded $(\mathfrak{a}, \mathfrak{b})$-bimodule, $a \mathrm{Z}$-graded $(\mathfrak{b}, \mathfrak{c})$-bimodule and $a \mathrm{Z}$-graded left a-module respectively. Then, the following isomorphism of Z-graded left c-modules holds:

$$
\mathscr{H} \operatorname{om}_{\mathfrak{a}}\left(L \otimes_{\mathfrak{b}} M, N\right) \simeq \mathscr{H} \mathrm{om}_{\mathfrak{b}}\left(M, \mathscr{H} \mathrm{om}_{\mathfrak{a}}(L, N)\right)
$$

As a corollary of this lemma, we have

COROLlary 3.1. Let a and c be Z-graded Lie superalgebras, and let $\$$ be a Zgraded subalgebra of a. Suppose that $M$ and $N$ are a $Z$-graded $(\mathfrak{s}, \mathfrak{c})$-bimodule and a Z-graded left a-module respectively. There exists an isomorphism of Zgraded left c-modules

$$
\mathscr{H} \operatorname{om}_{\mathfrak{a}}\left(\operatorname{Ind}_{\mathfrak{g}}^{\mathfrak{a}} M, N\right) \simeq \mathscr{H}_{\mathrm{om}}\left(M, \operatorname{Res}_{\mathfrak{g}}^{\mathfrak{a}} N\right) .
$$

\subsection{Definition}

Let $a$ be a Lie superalgebra, and let $U(\mathfrak{a})$ be the universal enveloping algebra of a. For $x \in \mathfrak{a}$, we define $L_{x}$ and $R_{x} \in \operatorname{End}_{\mathrm{K}}(U(\mathfrak{a}))$ by

$$
L_{x}(a):=x a, \quad R_{x}(a):=(-1)^{|x||a|} a x .
$$

Note that ad $x \in \operatorname{End}_{\mathrm{K}}(U(\mathfrak{a}))$ can be expressed as ad $x=L_{x}-R_{x}$.

$U(\mathfrak{n})$ has the natural $(\mathfrak{n}, \mathfrak{n})$-bimodule structure, and we regard

$$
U(\mathfrak{n})^{\circledast}:=\mathscr{H}_{\mathrm{om}}\left(U(\mathfrak{n}), \mathrm{K}_{\frac{0}{0}}^{0}\right)
$$

as $(\mathfrak{n}, \mathfrak{n})$-bimodule via (10) and (11).

Remark that the universal enveloping algebra $U(\mathfrak{n})$ is naturally $\mathbf{Z}_{\leq 0}$-graded associative algebra, and under the assumption that $\operatorname{dim} \mathrm{g}^{i}<\infty\left(i \in \mathbf{Z}_{<0}\right)$, each graded component $U(\mathfrak{n})^{i}$ is finite dimensional. Hence, $U(\mathfrak{n})^{\circledast}$ is a $Z_{\geq 0}$-graded $(\mathfrak{n}, \mathfrak{n})$-bimodule whose homogeneous components are finite dimensional.

Let $\gamma$ be a semi-infinite character of $\mathfrak{g}$, and let $\mathbf{K}_{-\gamma}:=\mathbf{K} \mathbf{1}_{-\gamma}$ be the one dimensional $Z_{2} \times \mathbf{Z}$-graded left $\mathfrak{b}$-module defined by

(1) $\operatorname{dim}\left(\mathrm{K}_{-\gamma}\right)_{\sigma}^{i}=\left\{\begin{array}{ll}1 & (\sigma, i)=(\overline{0}, 0) \\ 0 & (\sigma, i) \neq(\overline{0}, 0)\end{array}\right.$,

(2) $h . \mathbf{1}_{-\gamma}=-\gamma(h) \mathbf{1}_{-\gamma} \quad(h \in \mathfrak{h})$,

(3) $x \cdot \mathbf{1}_{-\gamma}=0 \quad\left(x \in \mathfrak{g}^{+}\right)$. 
Definition 3.1. We set

$$
S_{\gamma}(\mathfrak{g}):=U(\mathfrak{n})^{\circledast} \otimes_{\mathrm{K}} U(\mathfrak{b})
$$

and introduce a $(\mathfrak{g}, \mathfrak{g})$-bimodule structure on $S_{\gamma}(\mathfrak{g})$ as follows:

The left g-action on $S_{\gamma}(\mathrm{g})$ is defined via the following isomorphisms of $\mathrm{Z}_{2} \times \mathrm{Z}$-graded $\mathrm{K}$-vector spaces.

$$
\begin{aligned}
& i_{1}: S_{\gamma}(\mathfrak{g}) \stackrel{\sim}{\longrightarrow} \mathscr{H} \operatorname{om}_{\mathrm{K}}(U(\mathfrak{n}), U(\mathfrak{b})), \\
& i_{2}: \mathscr{H}_{\mathrm{om}_{\mathfrak{b}}}\left(U(\mathfrak{g}), \mathrm{K}_{-\gamma} \otimes_{\mathrm{K}} U(\mathfrak{b})\right) \stackrel{\sim}{\longrightarrow} \mathscr{H}_{\mathrm{om}_{\mathrm{K}}}(U(\mathfrak{n}), U(\mathfrak{b})) .
\end{aligned}
$$

Here, the first isomorphism $i_{1}$ is defined by

$$
i_{1}(\phi \otimes b)(u):=(-1)^{|b||u|} \phi(u) b \quad(u \in U(\mathfrak{n}))
$$

for $\phi \in U(\mathfrak{n})^{\circledast}$ and $b \in U(\mathfrak{b})$, and the second isomorphism $i_{2}$ is defined by

$$
i_{2}(\psi):=\left.i_{3} \circ \psi\right|_{U(\mathfrak{n})}
$$

where $\psi \in \mathscr{H}_{\mathrm{om}}\left(U(\mathfrak{g}), \mathrm{K}_{-\gamma} \otimes_{\mathrm{K}} U(\mathfrak{b})\right)$ and

$$
i_{3}: \mathrm{K}_{-\gamma} \otimes_{\mathrm{K}} U(\mathfrak{b}) \rightarrow U(\mathfrak{b})
$$

is defined by $\mathbf{1}_{-\gamma} \otimes b \mapsto b(b \in U(\mathfrak{b}))$. By regarding $\mathscr{l} \mathrm{om}_{\mathfrak{b}}\left(U(\mathfrak{g}), \mathrm{K}_{-\gamma} \otimes_{\mathrm{K}}\right.$ $U(\mathfrak{b}))$ as a left $\mathfrak{g}$-module as in Subsection 3.1, we introduce the left $\mathfrak{g}$-module structure on $S_{\gamma}(\mathfrak{g})$ via the isomorphisms $i_{1}$ and $i_{2}$.

The right action on $S_{\gamma}(\mathfrak{g})$ is defined via the following isomorphism of $\mathrm{Z}_{2} \times \mathrm{Z}$-graded $\mathrm{K}$-vector spaces:

$$
S_{\gamma}(\mathfrak{g}) \stackrel{\sim}{\longrightarrow} U(\mathfrak{n})^{\circledast} \otimes_{\mathfrak{n}} U(\mathfrak{g}) .
$$

By regarding $U(\mathfrak{n})^{\circledast} \otimes_{\mathfrak{n}} U(\mathfrak{g})$ as a right $\mathfrak{g}$-module via the right multiplication, we introduce the right $\mathfrak{g}$-module structure on $S_{\gamma}(\mathfrak{g})$.

Let us describe these actions explicitly. First, we introduce some notation. Let $\left\{x_{k}\right\}_{k \in I^{+}},\left\{h_{k}\right\}_{k \in I^{0}}$ and $\left\{y_{k}\right\}_{k \in I^{-}}$be bases of $\mathfrak{g}^{+}, \mathfrak{h}$ and $\mathfrak{n}$ in (6). By the isomorphism $U(\mathfrak{g}) \simeq U(\mathfrak{b}) \otimes_{\mathrm{K}} U(\mathfrak{n})$, for each $e \in \mathfrak{g}^{+}$and $u \in U(\mathfrak{n})$, there uniquely exist $H_{e}^{k}(u) \in U(\mathfrak{n})\left(k \in I^{0}\right), X_{e}^{k}(u) \in U(\mathfrak{n})\left(k \in I^{+}\right)$and $N_{e}(u) \in$ $U(\mathfrak{n})$ such that

$$
[u, e]=\sum_{k \in I^{0}} h_{k} H_{e}^{k}(u)+\sum_{k \in I^{+}} x_{k} X_{e}^{k}(u)+N_{e}(u) .
$$


Let us denote the linear maps

$$
u \longmapsto H_{e}^{k}(u), \quad u \longmapsto X_{e}^{k}(u), \quad u \longmapsto N_{e}(u) \quad(u \in U(\mathfrak{n}))
$$

by $H_{e}^{k}, X_{e}^{k}$ and $N_{e}$ respectively.

On the other hand, the isomorphism $U(\mathfrak{g}) \simeq U(\mathfrak{n}) \otimes_{\mathrm{K}} U(\mathfrak{b})$ implies that, for each $f \in \mathfrak{n}$ and $b \in U(\mathfrak{b})$, there uniquely exist $Y_{f}^{k}(b) \in U(\mathfrak{b})\left(k \in I^{-}\right)$ and $B_{f}(b) \in U(\mathfrak{b})$ such that

$$
[b, f]=\sum_{k \in I^{-}} y_{k} Y_{f}^{k}(b)+B_{f}(b) .
$$

Since we do not assume that $\mathfrak{g}$ is generated by its local part $\mathfrak{g}^{-1} \oplus \mathfrak{h} \oplus \mathfrak{g}^{1}$, the formulae in the following lemma and the proof of Theorem 3.1 look more complicate than in [12] and [2].

Lemma 3.2. For each $h \in \mathfrak{h}, e \in \mathfrak{g}^{+}, f \in \mathfrak{n}, \phi \in U(\mathfrak{n})^{\circledast}$ and $b \in U(\mathfrak{b})$, the following hold.

The left g-action:

$$
\begin{aligned}
h .(\phi \otimes b)= & (-1)^{|\phi||h|}\{\phi \otimes(-\gamma(h)+h) b-\phi \circ \text { ad } h \otimes b\}, \\
e .(\phi \otimes b)=( & -1)^{|\phi||e|} \phi \otimes e b \\
& +(-1)^{|e|}\left\{\sum_{k \in I^{0}}(-1)^{\left|h_{k}\right|} \phi \circ H_{e}^{k} \otimes\left(-\gamma\left(h_{k}\right)+h_{k}\right) b\right. \\
& \left.+\sum_{k \in I^{+}}(-1)^{\left|x_{k}\right|} \phi \circ X_{e}^{k} \otimes x_{k} b+\phi \circ N_{e} \otimes b\right\},
\end{aligned}
$$

$f .(\phi \otimes b)=(-1)^{|\phi||f|} \phi \circ R_{f} \otimes b$,

The right $\mathrm{g}$-action:

$(\phi \otimes b) \cdot h=\phi \otimes(b h)$,

$(\phi \otimes b) . e=\phi \otimes(b e)$,

$(\phi \otimes b) . f=(-1)^{|b||f|} \phi \circ L_{f} \otimes b+\sum_{k \in I^{-}} \phi \circ L_{y_{k}} \otimes Y_{f}^{k}(b)+\phi \otimes B_{f}(b)$.

Combining these formulae with Lemma 2.2, one can check the following theorem in a way similar to Theorem 1.3 in [12].

THEOREM 3.1. $S_{\gamma}(\mathfrak{g})$ is a $(\mathfrak{g}, \mathfrak{g})$-bimodule, i.e., the left action and the right action commute. 
Remark that, by definition, $S_{\gamma}(\mathfrak{g})$ is a $\mathrm{Z}_{\geq 0}$-graded module.

We introduce some notation. Let $\mathbf{i}: U(\mathfrak{n})^{\circledast} \rightarrow S_{\gamma}(\mathfrak{g})$ be the map defined by $\varphi \mapsto \varphi \otimes 1 \in S_{\gamma}(\mathfrak{g})$ for $\varphi \in U(\mathfrak{n})^{\circledast}$, and let $\mathbf{i}_{L}$ and $\mathbf{i}_{R}$ be the maps defined by

$$
\begin{aligned}
\mathbf{i}_{L}: U(\mathfrak{g}) \otimes_{\mathfrak{n}} U(\mathfrak{n})^{\circledast} \longrightarrow S_{\gamma}(\mathrm{g}) & u \otimes \varphi \longmapsto u . \mathbf{i}(\varphi), \\
\mathbf{i}_{R}: U(\mathfrak{n})^{\circledast} \otimes_{\mathfrak{n}} U(\mathrm{~g}) \longrightarrow S_{\gamma}(\mathfrak{g}) & \varphi \otimes u \longmapsto \mathbf{i}(\varphi) . u,
\end{aligned}
$$

where $u \in U(\mathfrak{g})$. Then, we have

THEOREM 3.2.

(1) $\mathbf{i}_{L}$ is an isomorphism of $(\mathfrak{g}, \mathfrak{n})$-bimodules.

(2) $\mathbf{i}_{R}$ is an isomorphism of $(\mathfrak{n}, \mathfrak{g})$-bimodules.

Proof. By using Lemma 3.2, we can directly show that $\mathbf{i}_{L}$ (resp. $\mathbf{i}_{R}$ ) is a homomorphism of $(\mathfrak{g}, \mathfrak{n})$-bimodules (resp. ( $\mathfrak{n}, \mathfrak{g})$-bimodules). Moreover, by the definition of $S_{\gamma}(\mathrm{g}), \mathbf{i}_{R}$ is a bijection. Hence, we have to show that $\mathbf{i}_{L}$ is a bijection. To prove the bijectivity, we use a triangularity of the left $\mathfrak{b}$-action on $S_{\gamma}(\mathfrak{g})$. To state the triangularity, we introduce a filtration of $S_{\gamma}(\mathfrak{g})$

We first introduce filtrations of $U(\mathfrak{b})$. Let $\left\{F_{i} U(\mathfrak{b}) \mid i \in \mathbf{Z}_{\geq 0}\right\}$ be a standard filtration of $U(\mathfrak{b})$, and let $\left\{F_{i}^{j} U(\mathfrak{b}) \mid i \in \mathbf{Z}_{>0}, j \in \mathbf{Z}_{\geq-1}\right\}$ be the refinement of $\left\{F_{i} U(\mathfrak{b}) \mid i \in \mathbf{Z}_{\geq 0}\right\}$ defined by

$$
F_{i}^{j} U(\mathfrak{b}):=\left\{\begin{array}{ll}
\mathfrak{b}^{\leq j} F_{i-1} U(\mathfrak{b})+F_{i-1} U(\mathfrak{b}) & (j \geq 0) \\
F_{i-1} U(\mathfrak{b}) & (j=-1)
\end{array} .\right.
$$

By definition, $F_{i-1} U(\mathfrak{b}) \subset F_{i}^{0} U(\mathfrak{b}) \subset F_{i}^{1} U(\mathfrak{b}) \subset \cdots \subset F_{i} U(\mathfrak{b})$ and $\bigcup_{j=-1}^{\infty} F_{i}^{j} U(\mathfrak{b})=F_{i} U(\mathfrak{b})$. Note that for $j \geq 0$,

$$
F_{i}^{j} U(\mathfrak{b}) / F_{i}^{j-1} U(\mathfrak{b}) \simeq \mathfrak{b}^{j} S^{i-1}\left(\mathfrak{b}^{\geq j}\right) \subset S^{i}(\mathfrak{b}),
$$

where $S^{i}(\mathfrak{b})$ denotes the $i$ th homogeneous component of the symmetric algebra $S(\mathfrak{b})$ on $\mathfrak{b}$.

We further set

$$
F_{i}^{j} S_{\gamma}(\mathfrak{g}):=U(\mathfrak{n})^{\circledast} \otimes_{\mathrm{K}} F_{i}^{j} U(\mathfrak{b}) .
$$

Then, by Lemma 3.2, for $i \in \mathbf{Z}_{>0}, j, k \in \mathbf{Z}_{\geq 0}, b \in F_{i-1}^{j} U(\mathfrak{b}) \backslash F_{i-1}^{j-1} U(\mathfrak{b})$, $b_{1} \in \mathfrak{b}^{k}$ and $\varphi \in U(\mathfrak{n})^{\circledast}$, we have $b_{1} \cdot(\varphi \otimes b) \in F_{i}^{\min \{j, k\}} S_{\gamma}(\mathfrak{g})$ and

$$
b_{1} \cdot(\varphi \otimes b) \equiv(-1)^{\left|b_{1}\right||\varphi|} \varphi \otimes b_{1} b \quad\left(\bmod F_{i}^{\min \{j, k\}-1} S_{\gamma}(\mathfrak{g})\right) .
$$

Using this formula, one can easily prove that $\iota_{L}$ is bijective. 


\section{Feigin-Arkhipov-Soergel duality}

By means of the semi-regular bimodule, we construct an equivalence of categories of Z-graded modules with finite $\Delta$-flags. The arguments in this section essentially follow from [12].

\subsection{Preliminaries}

As preliminary steps, here, we show two isomorphisms of left Z-graded nmodules.

For $u \in U(\mathfrak{n})$, let $\iota_{u}$ be the element of $\mathscr{H} \mathrm{om}_{\mathfrak{n}}\left(U(\mathfrak{n})^{\circledast}, U(\mathfrak{n})^{\circledast}\right)$ defined by

$$
\iota_{u}(\phi):=(-1)^{|\phi||u|} \phi \circ L_{u} \quad\left(\phi \in U(\mathfrak{n})^{\circledast}\right) .
$$

One can directly check that $\iota_{u}\left(u_{1} . \phi\right)=(-1)^{|u|\left|u_{1}\right|} u_{1} .\left(\iota_{u}(\phi)\right)$ for any $u_{1} \in$ $U(\mathfrak{n})$, and thus, $\iota_{u} \in \mathscr{H} 0 \mathrm{~m}_{\mathfrak{n}}\left(U(\mathfrak{n})^{\circledast}, U(\mathfrak{n})^{\circledast}\right)$.

LEMMA 4.1. The map

$$
\iota: U(\mathfrak{n}) \rightarrow \mathscr{H}^{\circ} \mathrm{m}_{\mathfrak{n}}\left(U(\mathfrak{n})^{\circledast}, U(\mathfrak{n})^{\circledast}\right) \quad\left(u \longmapsto \iota_{u}\right)
$$

is an isomorphism of Z-graded left $\mathrm{n}$-modules.

Proof. By Lemma 3.1, the following isomorphisms of Z-graded left $\mathfrak{n}-$ modules hold.

$$
\begin{aligned}
\mathscr{H o m}_{\mathfrak{n}}\left(U(\mathfrak{n})^{\circledast}, U(\mathfrak{n})^{\circledast}\right)=\mathscr{H} \mathrm{om}_{\mathfrak{n}}\left(U(\mathfrak{n})^{\circledast}, \mathscr{H} \mathrm{om}_{\mathrm{K}}\left(U(\mathfrak{n}), \mathrm{K}_{\overline{0}}^{0}\right)\right) \\
\simeq \mathscr{H} \mathrm{om}_{\mathrm{K}}\left(U(\mathfrak{n}) \otimes_{\mathfrak{n}} U(\mathfrak{n})^{\circledast}, \mathrm{K}_{\overline{0}}^{0}\right) \simeq \mathscr{H} \mathrm{om}_{\mathrm{K}}\left(U(\mathfrak{n})^{\circledast}, \mathrm{K}_{\overline{0}}^{0}\right) \simeq U(\mathfrak{n}) .
\end{aligned}
$$

Recall that the antipode $a$ of a Lie superalgebra $a$ is defined by

$$
\begin{aligned}
a(x) & :=-(-1)^{|x|} x \quad(x \in \mathfrak{a}), \\
a\left(u_{1} u_{2}\right) & :=(-1)^{\left|u_{1}\right|\left|u_{2}\right|} a\left(u_{2}\right) a\left(u_{1}\right) \quad\left(u_{1}, u_{2} \in U(\mathfrak{a})\right) .
\end{aligned}
$$

For a $Z_{2} \times Z$-graded module $V$ over a Z-graded Lie superalgebra a, let $V^{\sharp a}:=$ $\mathscr{H} \mathrm{m}_{\mathrm{K}}\left(V, \mathrm{~K}_{\overline{0}}^{0}\right)$ be the antipode dual of $V$ whose $\mathrm{g}$-module structure is given by

$$
(u . \phi)(v):=(-1)^{|u||\phi|} \phi(a(u) . v) \quad\left(u \in U(\mathfrak{a}), \phi \in V^{\sharp a}, v \in V\right) .
$$

Notice that $U(\mathfrak{n})^{\sharp a}=U(\mathfrak{n})^{\circledast}$ as K-vector space. Let ${ }^{t} a: U(\mathfrak{n})^{\circledast} \rightarrow U(\mathfrak{n})^{\sharp a}$ be the transpose of the antipode $a: U(\mathfrak{n}) \rightarrow U(\mathfrak{n})$.

LEMma 4.2. There is the following isomorphism of Z-graded left $\mathfrak{n}$-modules

$$
{ }^{t} a: U(\mathfrak{n})^{\circledast} \simeq U(\mathfrak{n})^{\sharp a} .
$$


4.2. Category of $\mathrm{g}$-modules with finite $\Delta$-flags

Let $\operatorname{Mod}_{\mathfrak{g}}^{Z}$ be the category of the $Z_{2} \times Z$-graded left $g$-modules whose morphisms are given by

$$
\operatorname{Hom}_{\operatorname{Mod}_{\mathfrak{g}}^{z}}(M, N):=\operatorname{Hom}_{\mathfrak{g}}(M, N)_{\overline{0}}^{0} .
$$

Definition 4.1. We define the full subcategories $\mathcal{M}$ and $\mathscr{K}$ of $\operatorname{Mod}_{\mathfrak{g}}^{Z}$ as follows:

(1) $M \in \mathrm{Ob}(\mathscr{M}) \Leftrightarrow \operatorname{Res}_{\mathfrak{n}}^{\mathfrak{g}} M \simeq U(\mathfrak{n}) \otimes_{\mathrm{K}} E$ for some finite dimensional $\mathrm{Z}_{2} \times \mathrm{Z}$-graded K-vector space $E$,

(2) $K \in \mathrm{Ob}(\mathscr{K}) \Leftrightarrow \operatorname{Res}_{\mathfrak{n}}^{\mathfrak{g}} K \simeq U(\mathfrak{n})^{\circledast} \otimes_{\mathrm{K}} E$ for some finite dimensional $\mathrm{Z}_{2} \times \mathrm{Z}$-graded K-vector space $E$.

The categories $\mathscr{M}$ and $\mathscr{K}$ are additive categories, and they are not, in general, abelian categories.

For $M \in \mathrm{Ob}(\mathscr{M})$ such that $\operatorname{Res}_{\mathfrak{n}}^{\mathfrak{g}} M \simeq U(\mathfrak{n}) \otimes_{\mathrm{K}} E$, where $E$ is a $\mathrm{Z}_{2} \times \mathrm{Z}$ graded K-vector space, we define the rank of $M$ by $\operatorname{dim} E$, and denote it by rk $M$. Since $\operatorname{ch} M$ defined in (1) is expressed as $\operatorname{ch} M=\operatorname{ch} U(\mathfrak{n}) \operatorname{ch} E$, the rank of $M$ is well-defined. We similarly define rk $K$ for $K \in \mathrm{Ob}(\mathscr{K})$.

Here, we state a characterization of the category $\mathcal{M}$. We first introduce an object of $\mathcal{M}$.

Let $E$ be a finite dimensional $\mathrm{Z}_{2} \times \mathrm{Z}$-graded left $\mathfrak{h}$-module. We regard $E$ as left $\mathfrak{b}$-module via $\left.\mathrm{g}^{+}\right|_{E} \equiv 0$, and set

$$
\Delta(E):=U(\mathfrak{g}) \otimes_{\mathfrak{b}} E .
$$

By definition, $\Delta(E) \in \mathrm{Ob}(\mathscr{M})$.

Suppose that $M(\neq\{0\})$ is a Z-graded left $g$-module. A sequence of Z-graded $\mathrm{g}$-submodules of finite length

$$
\{0\}=M(0) \subset M(1) \subset M(2) \subset \cdots \subset M(l)=M
$$

is called a finite $\Delta$-flag of $M$ if for each $k \in Z_{>0}$, there exists an irreducible finite dimensional $Z_{2} \times Z$-graded $\mathfrak{h}$-module $E_{k}$ such that $M(k) / M(k-1) \simeq \Delta\left(E_{k}\right)$.

Proposition 4.1. $M \in \mathrm{Ob}(\mathcal{M})$ if and only if $M=\{0\}$ or $M$ has a finite $\Delta$-flag. Moreover, any $\Delta$-flag $M(0) \subset M(1) \subset M(2) \subset \cdots \subset M(l)$ of $M \in \mathrm{Ob}(\mathcal{M})$ satisfies that $M(k) \in \mathrm{Ob}(\mathcal{M})$ for all $k$.

Proof. ("if" part) Suppose that $M \in \mathrm{Ob}\left(\operatorname{Mod}_{\mathrm{g}}^{\mathrm{Z}}\right)$ has a finite $\Delta$-flag. Let

$$
\{0\}=M(0) \subset M(1) \subset M(2) \subset \cdots \subset M(l)=M
$$


be a finite $\Delta$-flag of $M$, and let $E_{k}$ be a $Z_{2} \times Z$-graded h-module such that $M(k) / M(k-1) \simeq \Delta\left(E_{k}\right)$. Since $0 \rightarrow M(k-1) \rightarrow M(k) \rightarrow \Delta\left(E_{k}\right) \rightarrow 0$ is exact and $\Delta\left(E_{k}\right)$ is $U(\mathfrak{n})$-free, we have

$$
\operatorname{Res}_{\mathfrak{n}}^{\mathfrak{g}} M(k) \simeq \operatorname{Res}_{\mathfrak{n}}^{\mathfrak{g}} M(k-1) \oplus \operatorname{Res}_{\mathfrak{n}}^{\mathfrak{g}} \Delta\left(E_{k}\right) .
$$

Hence, by induction on $k$, one can prove that $M(k) \in \operatorname{Ob}(\mathscr{M})$, and thus, $M \in \mathrm{Ob}(\mathscr{M})$.

("only if" part) We show the statement by induction on rk $M$. In the case where rk $M=1$, the assertion obviously holds. Suppose that $r:=$ rk $M>1$.

Let $\left\{u_{1}, \ldots, u_{r}\right\}$ be a $U(\mathfrak{n})$-basis of $\operatorname{Res}_{\mathfrak{n}}^{\mathfrak{g}} M$. We may assume that $u_{i}$ are homogeneous with respect to the $\mathrm{Z}_{2} \times \mathrm{Z}$-gradation. Set $F:=\bigoplus_{i=1}^{r} \mathrm{~K} u_{i}$. Let $m$ be the maximal integer such that $M^{m} \neq\{0\}$.

By definition, $M^{m} \subset F$. Since $M^{m}$ is a finite dimensional left $\mathfrak{h}$-module, there exists an irreducible finite dimensional left $\mathfrak{h}$-submodule $E$ of $M^{m}$. We set $N:=U(\mathfrak{g}) . E$. Then,

$$
N \simeq \Delta(E)
$$

as Z-graded left g-module. If $E=F$, then, $M=N$ and the assertion holds. If $E \subsetneq F$, then, we have

$$
\operatorname{Res}_{\mathfrak{n}}^{\mathfrak{g}}(M / N) \simeq U(\mathfrak{n}) \otimes_{\mathrm{K}}(F / E),
$$

and thus, $M / N \in \operatorname{Ob}(\mathscr{M})$. By the induction hypothesis, $M / N$ has a finite $\Delta$-flag. Hence, $M$ also has a finite $\Delta$-flag.

\subsection{Functors between $\mathcal{M}$ and $\mathscr{K}$}

We first introduce two functors. Let $S_{\gamma}(\mathfrak{g})$ be the semi-regular bimodule defined in (3.1).

DeFINITION 4.2.

(1) Define the functor $T: \mathscr{M} \rightarrow \mathscr{K}$ by

$$
T(M):=S_{\gamma}(\mathfrak{g}) \otimes_{\mathfrak{g}} M \quad(M \in \mathrm{Ob}(\mathscr{M})) .
$$

(2) Define the functor $H: \mathscr{K} \rightarrow \mathscr{M}$ by

$$
H(K):=\mathscr{H}_{\mathfrak{g}}\left(S_{\gamma}(\mathfrak{g}), K\right) \quad(K \in \mathrm{Ob}(\mathscr{K})) .
$$

Indeed, these functors are well-defined, i.e., the following holds.

Lemma 4.3. $T(M) \in \mathrm{Ob}(\mathscr{K})$ (resp. $H(K) \in \mathrm{Ob}(\mathscr{M}))$ for $M \in \mathrm{Ob}(\mathscr{M})$ (resp. $K \in \mathrm{Ob}(\mathscr{K})$ ). 
Proof. Let $E$ be a $Z_{2} \times Z$-graded $\mathrm{K}$-vector space such that $\operatorname{Res}_{\mathfrak{n}}^{\mathfrak{g}} M \simeq$ $U(\mathfrak{n}) \otimes_{\mathrm{K}} E$. By the isomorphism (13), we have

$$
\operatorname{Res}_{\mathfrak{n}}^{\mathfrak{g}} T(M) \simeq U(\mathfrak{n})^{\circledast} \otimes_{\mathrm{K}} E
$$

as Z-graded left n-module. Hence, $T(M) \in \mathrm{Ob}(\mathscr{K})$.

Next, let $E$ be a $Z_{2} \times Z$-graded $\mathrm{K}$-vector space such that $\operatorname{Res}_{\mathfrak{n}}^{\mathfrak{g}} K \simeq U(\mathfrak{n})^{\circledast} \otimes_{\mathrm{K}}$ $E$. By the isomorphism (12), Corollary 3.1 and Lemma 4.1, we have

$$
\begin{aligned}
\operatorname{Res}_{\mathfrak{n}}^{\mathfrak{g}} H(K) & \simeq \mathscr{H} \mathrm{om}_{\mathfrak{n}}\left(U(\mathfrak{n})^{\circledast}, \operatorname{Res}_{\mathfrak{n}}^{\mathfrak{g}} K\right) \\
& \simeq \mathscr{H}_{\mathrm{om}_{\mathfrak{n}}}\left(U(\mathfrak{n})^{\circledast}, U(\mathfrak{n})^{\circledast}\right) \otimes_{\mathrm{K}} E \\
& \simeq U(\mathfrak{n}) \otimes_{\mathrm{K}} E .
\end{aligned}
$$

Hence, $H(K) \in \mathrm{Ob}(\mathcal{M})$.

Lemma 4.2 implies the following lemma.

Lemma 4.4. For $M \in \mathrm{Ob}(\mathscr{M})$ (resp. $K \in \mathrm{Ob}(\mathscr{K}))$, we have $M^{\sharp a} \in \mathrm{Ob}(\mathscr{K})$ (resp. $K^{\sharp a} \in \mathrm{Ob}(\mathscr{M})$ ).

Proposition 4.2. The functors $T$ and $H$ send a short exact sequence to a short exact sequence.

Proof. We first show the statement for $T$. Let $M_{i}(i=1,2,3)$ be objects of $\mathcal{M}$ such that $0 \rightarrow M_{1} \rightarrow M_{2} \rightarrow M_{3} \rightarrow 0$ is an exact sequence of left g-modules. We show that

$$
0 \longrightarrow T\left(M_{1}\right) \longrightarrow T\left(M_{2}\right) \longrightarrow T\left(M_{3}\right) \longrightarrow 0
$$

is an exact sequence of left $\mathrm{g}$-modules.

Since $M_{3}$ is $U(\mathfrak{n})$-free, the sequence $0 \rightarrow \operatorname{Res}_{\mathfrak{n}}^{\mathfrak{g}} M_{1} \rightarrow \operatorname{Res}_{\mathfrak{n}}^{\mathfrak{g}} M_{2} \rightarrow$ $\operatorname{Res}_{\mathfrak{n}}^{\mathfrak{g}} M_{3} \rightarrow 0$ splits. Hence,

$0 \rightarrow U(\mathfrak{n})^{\circledast} \otimes_{\mathfrak{n}} \operatorname{Res}_{\mathfrak{n}}^{\mathfrak{g}} M_{1} \rightarrow U(\mathfrak{n})^{\circledast} \otimes_{\mathfrak{n}} \operatorname{Res}_{\mathfrak{n}}^{\mathfrak{g}} M_{2} \rightarrow U(\mathfrak{n})^{\circledast} \otimes_{\mathfrak{n}} \operatorname{Res}_{\mathfrak{n}}^{\mathfrak{g}} M_{3} \rightarrow 0$

also splits, and thus, it is an exact sequence of left n-modules. This means that the sequence (15) is exact, since $\operatorname{Res}_{\mathfrak{n}}^{\mathfrak{g}} T(M) \simeq U(\mathfrak{n})^{\circledast} \otimes_{\mathfrak{n}} \operatorname{Res}_{\mathfrak{n}}^{\mathfrak{g}} M$ for $M \in \mathrm{Ob}(\mathcal{M})$.

Next, we show the statement for $H$. Let $K_{i}(i=1,2,3)$ be objects of $\mathscr{K}$ such that $0 \rightarrow K_{1} \rightarrow K_{2} \rightarrow K_{3} \rightarrow 0$ is an exact sequence of g-modules. Since $K_{1}^{\sharp a}$ is $U(\mathfrak{n})$-free by Lemma 4.4 , taking the anti-pode dual, we see that the sequence

$$
0 \rightarrow \operatorname{Res}_{\mathfrak{n}}^{\mathfrak{g}} K_{1} \rightarrow \operatorname{Res}_{\mathfrak{n}}^{\mathfrak{g}} K_{2} \rightarrow \operatorname{Res}_{\mathfrak{n}}^{\mathfrak{g}} K_{3} \rightarrow 0
$$


splits. Since $\operatorname{Res}_{\mathfrak{n}}^{\mathfrak{g}} H(K) \simeq \mathscr{H} \mathrm{om}_{\mathfrak{l}}\left(U(\mathfrak{n})^{\circledast}, \operatorname{Res}_{\mathfrak{l}}^{\mathfrak{g}} K\right)$ for $K \in \mathrm{Ob}(\mathscr{K})$, by an argument similar to the above, one can prove the statement for $H$.

\subsection{Categorical equivalences}

Theorem 4.1. The functor $T: \mathscr{M} \rightarrow \mathscr{K}$ gives a categorical equivalence.

Proof. By Lemma 3.1, as K-vector space,

$$
\operatorname{Hom}_{\mathscr{K}}(T(M), K) \simeq \operatorname{Hom}_{\mathcal{M}}(M, H(K)),
$$

where $M \in \mathrm{Ob}(\mathcal{M})$ and $K \in \mathrm{Ob}(\mathscr{K})$. In fact, restricting the isomorphism

$$
\mathscr{H} \mathrm{om}_{\mathfrak{g}}\left(S_{\gamma}(\mathfrak{g}) \otimes_{\mathfrak{g}} M, K\right) \simeq \mathscr{H} \mathrm{om}_{\mathfrak{g}}\left(M, \mathscr{C} \mathrm{om}_{\mathfrak{g}}\left(S_{\gamma}(\mathfrak{g}), K\right)\right)
$$

to a homogeneous subspace, we obtain the isomorphism (16). Hence, $(T, H)$ is an adjoint pair, and thus, there exist natural transformations $\eta: \operatorname{id}_{\mathcal{M}} \Rightarrow H \circ T$ and $\epsilon: T \circ H \Rightarrow \operatorname{id}_{\mathscr{\epsilon}}$.

For each $M \in \mathrm{Ob}(\mathcal{M})$, we show that $\eta_{M}: M \rightarrow H \circ T(M)$ is an isomorphism. Let $E$ be a finite dimensional $Z_{2} \times Z$-graded $\mathrm{K}$-vector space such that $\operatorname{Res}_{\mathfrak{n}}^{\mathfrak{g}} M \simeq U(\mathfrak{n}) \otimes_{\mathrm{K}} E$. By Corollary 3.1, Theorem 3.2 and Lemma 4.1, we have

$$
\operatorname{Res}_{\mathfrak{n}}^{\mathfrak{g}} H \circ T(M) \simeq \mathscr{H} \mathrm{om}_{\mathfrak{n}}\left(U(\mathfrak{n})^{\circledast}, U(\mathfrak{n})^{\circledast}\right) \otimes_{\mathrm{K}} E \simeq U(\mathfrak{n}) \otimes_{\mathfrak{K}} E \simeq \operatorname{Res}_{\mathfrak{n}}^{\mathfrak{g}} M
$$

as Z-graded left $\mathfrak{n}$-module, and $\eta_{M}$ is this isomorphism of Z-graded left $\mathrm{g}$ modules.

One can similarly show that $\epsilon_{K}: T \circ H(K) \rightarrow K$ is an isomorphism of Z-graded left $\mathrm{g}$-modules for any $K \in \mathrm{Ob}(\mathscr{K})$. Hence, $H \circ T \simeq \mathrm{id}_{\mathscr{M}}$ and $T \circ H \simeq \operatorname{id}_{\mathscr{K}}$, i.e., $T$ and $H$ are quasi-inverse to each other, and thus, $T$ is a categorical equivalence.

We remark that, by Lemma $4.4,(\cdot)^{\sharp a}$ defines a contravariant functor from $\mathscr{K}$ to $\mathscr{M}$. Hence, it defines a covariant functor from $\mathscr{K}$ to $\mathscr{M}^{\text {op }}$, where $\mathscr{M}^{\text {op }}$ denotes the opposite category of $\mathscr{M}$.

Proposition 4.3. The covariant functor $(\cdot)^{\sharp a}: \mathscr{K} \rightarrow \mathscr{M}^{\text {op }}$ defines a categorical equivalence, which sends a short exact sequence to a short exact sequence.

Finally, we define a functor $\Phi$ as follows:

$$
\Phi: \mathscr{M} \longrightarrow \mathscr{M}^{\mathrm{op}} \quad\left(M \longmapsto T(M)^{\sharp a}\right) .
$$

Here, we call the following equivalence Feigin-Arkhipov-Soergel duality. 
THEOREM 4.2. The covariant functor $\Phi: \mathcal{M} \rightarrow \mathscr{M}^{\mathrm{op}}$ defines a categorical equivalence, and it sends a short exact sequence to a short exact sequence.

Proof. By Proposition 4.2, Theorem 4.1 and Proposition 4.3, we obtain the theorem.

Moreover, we have

Proposition 4.4. Let $E$ be an irreducible finite dimensional $Z_{2} \times Z$-graded left $\mathfrak{h}$-module. Then, the following isomorphism of Z-graded left $\mathrm{g}$-modules holds:

$$
\Phi(\Delta(E)) \cong \Delta\left(\mathrm{K}_{\gamma} \otimes E^{\sharp a}\right) .
$$

Proof. Since $E$ is irreducible, there exists $t \in \mathrm{Z}$ such that $E^{t}=E$. The isomorphism

$$
T(\Delta(E)) \simeq S_{\gamma}(\mathfrak{g}) \otimes_{\mathfrak{b}} E \simeq \mathscr{H}^{\circ} \mathrm{m}_{\mathfrak{b}}\left(U(\mathfrak{g}), \mathrm{K}_{-\gamma} \otimes_{\mathfrak{K}} U(\mathfrak{b})\right) \otimes_{\mathfrak{b}} E
$$

of left g-module implies that $T(\Delta(E))^{t} \simeq \mathrm{K}_{-\gamma} \otimes_{\mathrm{K}} E$ as left $\mathfrak{h}$-module, and $T(\Delta(E))^{j}=\{0\}$ for $j<t$. Thus, $\Phi(\Delta(E))^{-t} \simeq \mathrm{K}_{\gamma} \otimes_{\mathrm{K}} E^{\sharp a}$ as left $\mathfrak{h}$-module, and $T(\Delta(E))^{j}=\{0\}$ for $j>-t$. Hence,

$$
\Phi(\Delta(E))^{\geq-t} \simeq \mathrm{K}_{\gamma} \otimes_{\mathrm{K}} E^{\sharp a}
$$

as Z-graded left $\mathfrak{b}$-module, and thus, there exists a homomorphism

$$
\Delta\left(\mathrm{K}_{\gamma} \otimes_{\mathrm{K}} E^{\sharp a}\right) \longrightarrow \Phi(\Delta(E))
$$

of Z-graded left $\mathrm{g}$-modules.

On the other hand, by the isomorphism $\operatorname{Res}_{\mathfrak{n}}^{\mathfrak{g}} T(\Delta(E)) \simeq U(\mathfrak{n})^{\circledast} \otimes_{\mathrm{K}} E$ and Lemma 4.2, we have

$$
\operatorname{Res}_{\mathfrak{n}}^{\mathfrak{g}} \Phi(\Delta(E)) \simeq U(\mathfrak{n}) \otimes_{\mathfrak{K}} E^{\sharp a}
$$

as Z-graded left $\mathfrak{n}$-module. Hence, (19) is an isomorphism of Z-graded left $\mathfrak{n}$-modules, i.e., an isomorphism of Z-graded left g-modules.

\section{Semi-infinite characters of superconformal algebras}

In order to describe the Feigin-Arkhipov-Soergel duality for superconformal algebras explicitly, we determine the semi-infinite characters of them. 


\subsection{Lie superalgebras of Cartan type}

In [8], V. Kac introduced a class of Z-graded Lie superalgebras called physical superconformal algebras, and classified them. According to his classification, the physical superconformal algebras are the Lie superalgebras of Cartan type.

In Section 5.1, we first give examples of Lie superalgebras of Cartan type, and next, we recall the definition and the classification of physical superconformal algebras.

5.1.1. Lie superalgebra $W(N)$. Let $\mathrm{C}\left[t, t^{-1}\right]$ be the ring of Laurent polynomials in a variable $t$, and $\Lambda(N)$ the Grassmann algebra generated by $N$ odd variables $\theta_{1}, \ldots, \theta_{N}$. Set $\Lambda_{t}(N):=\mathrm{C}\left[t, t^{-1}\right] \otimes_{\mathrm{C}} \Lambda(N)$. It naturally becomes a supercommutative superalgebra with $|t|=\overline{0}$ and $\left|\theta_{i}\right|=\overline{1}$. Recall that the Lie superalgebra $W(1, N)$ is defined by

$$
\begin{gathered}
W(1, N):=\operatorname{Der} \Lambda_{t}(N)=\left\{P_{0} \frac{\partial}{\partial t}+\sum_{i=1}^{N} P_{i} \frac{\partial}{\partial \theta_{i}} \mid P_{i} \in \Lambda_{t}(N)\right\}, \\
\left|\frac{\partial}{\partial t}\right|:=\overline{0},\left|\frac{\partial}{\partial \theta_{i}}\right|:=\overline{1} \text { and } \\
{\left[D_{1}, D_{2}\right]:=D_{1} \circ D_{2}-(-1)^{\left|D_{1}\right|\left|D_{2}\right|} D_{2} \circ D_{1} \quad\left(D_{1}, D_{2} \in W(1, N)\right) .}
\end{gathered}
$$

For simplicity, in the sequel, we denote $W(1, N)$ by $W(N)$.

To introduce Z-gradations of $W(N)$, we remark the following fact:

Lemma 5.1. For each $n \in \mathrm{Z}$, we set

$$
L_{n}:=-t^{n+1} \frac{\partial}{\partial t}-\sum_{i=1}^{N} a_{n}^{(i)} t^{n} \theta_{i} \frac{\partial}{\partial \theta_{i}} \quad\left(a_{n}^{(i)} \in \mathrm{C}\right) .
$$

Then, $\left[L_{m}, L_{n}\right]=(m-n) L_{m+n}$ holds for any $m, n \in \mathbf{Z}$ if and only if $a_{n}^{(i)}=$ $\alpha_{i} n+\beta_{i}$ for some constants $\alpha_{i}$ and $\beta_{i}$.

If $\beta_{i} \in \frac{1}{2} \mathrm{Z}$ for any $i$, then we have

$$
W(N)=\bigoplus_{m \in Z} W(N)^{m}, \quad W(N)^{m}:=\left\{x \in W(N) \mid\left[L_{0}, x\right]=-\frac{m}{2} x\right\} .
$$

Here, we call $W(N)$ with the Z-gradation defined by $\beta_{i}=\frac{1}{2}$ for any $i$ the Neveu-Schwarz sector (cf. [8] Corollary 2.1). We also refer the case $\beta_{i}=0$ for any $i$ as the Ramond sector.

It is known that $W(N)$ with the Z-gradation (20) is a simple Z-graded Lie superalgebra, i.e., it has no non-trivial Z-graded ideal. 
5.1.2. Lie superalgebra $S(N ; \alpha)$. For $\alpha \in \mathrm{C}$, the Lie superalgebra $S(N ; \alpha)$ is defined as follows:

$$
S(N ; \alpha):=\left\{D \in W(N) \mid \operatorname{div}\left(t^{\alpha} D\right)=0\right\},
$$

where for $P_{1}, \ldots, P_{N} \in \Lambda_{t}(N)$, we set

$$
\operatorname{div}\left(t^{\alpha} P_{0} \frac{\partial}{\partial t}+\sum_{i=1}^{N} t^{\alpha} P_{i} \frac{\partial}{\partial \theta_{i}}\right):=\frac{\partial\left(t^{\alpha} P_{0}\right)}{\partial t}+\sum_{i=1}^{N}(-1)^{\left|P_{i}\right|} \frac{\partial\left(t^{\alpha} P_{i}\right)}{\partial \theta_{i}} .
$$

Let $S^{\prime}(N ; \alpha)$ be the derived subalgebra of $S(N ; \alpha)$. Note that

$$
S(N ; \alpha)=\left\{\begin{array}{ll}
S^{\prime}(N ; \alpha) & \text { if } \alpha \notin \mathbf{Z} \\
S^{\prime}(N ; \alpha) \oplus \mathrm{C} t^{-\alpha} \theta_{1} \cdots \theta_{N} \frac{\partial}{\partial t} & \text { if } \alpha \in \mathbf{Z}
\end{array} .\right.
$$

REMARK 5.1. In [9], it was remarked that central extensions of $S^{\prime}(2 ; 0)$ and $S^{\prime}(2 ;-1)$ are respectively isomorphic (as Z-graded Lie superalgebra) to the Neveu-Schwarz and the Ramond sectors of the small $N=4$ superconformal algebras introduced in [13]. (For the commutation relations of the small $N=4$ superconformal algebras, see [4].)

In the sequel, we consider only the cases where $N=2$ and $\alpha=0,-1$. We call $S^{\prime}(2 ; 0)$ and $S^{\prime}(2 ;-1)$ the Neveu-Schwarz sector and the Ramond sector respectively.

Here, we consider the following Z-gradation of $S^{\prime}(2 ; \alpha)$. For simplicity, we set

$$
L_{n}:=-t^{n+1} \frac{\partial}{\partial t}-\frac{n+\alpha+1}{2} t^{n}\left(\theta_{1} \frac{\partial}{\partial \theta_{1}}+\theta_{2} \frac{\partial}{\partial \theta_{2}}\right) \quad(n \in \mathbf{Z}) .
$$

Then, $L_{n} \in S^{\prime}(2 ; \alpha)$ by definition, and $\left[L_{m}, L_{n}\right]=(m-n) L_{m+n}$ by Lemma 5.1. Since $\alpha \in\{0,-1\}$, we have

$$
S^{\prime}(2 ; \alpha)=\bigoplus_{m \in Z} S^{\prime}(2 ; \alpha)^{m}, \quad S^{\prime}(2 ; \alpha)^{m}:=\left\{x \in S^{\prime}(2 ; \alpha) \mid\left[L_{0}, x\right]=-\frac{m}{2} x\right\} .
$$

Remark that $S^{\prime}(2 ; \alpha)$ is a simple Z-graded subalgebra of $W(2)$ with Z-gradation (20) defined by $\beta_{1}=\beta_{2}=\frac{1+\alpha}{2}$.

5.1.3. Lie superalgebra $K(N ; \epsilon)$. Let

$$
\Omega^{1}:=\Lambda_{t}(N) d t \oplus \bigoplus_{i=1}^{N} \Lambda_{t}(N) d \theta_{i}
$$


be the $\left(\Lambda_{t}(N), \Lambda_{t}(N)\right)$-bimodule defined by $|d t|:=\overline{1},\left|d \theta_{i}\right|:=\overline{0}, g(f d x):=$ $(g f) d x$ and $(f d x) g:=(-1)^{|d x||g|}(f g) d x$ for $f, g \in \Lambda_{t}(N)$ and $x=t$ or $\theta_{i}$. We define a linear map

$$
d: \Lambda_{t}(N) \longrightarrow \Omega^{1}, \quad f \longmapsto(-1)^{|f|} \frac{\partial f}{\partial t} d t+\sum_{i=1}^{N} \frac{\partial f}{\partial \theta_{i}} d \theta_{i} .
$$

Then, the map $d$ satisfies $d(f g)=d(f) g+(-1)^{|f|} f d g . W(N)$ naturally acts on $\Omega^{1}$ as follows:

$$
D(f d g):=(D f) d g+(-1)^{|D||f|+|D|} f d(D g) .
$$

For each $\epsilon=\left(\epsilon_{i}\right)_{i=1}^{N} \in \mathbf{Z}^{\oplus N}$, the Z-graded Lie superalgebra $K(N ; \epsilon)$ is defined as follows:

$$
K(N ; \epsilon):=\left\{D \in W(N) \mid D \omega_{\epsilon}=P \omega_{\epsilon}\left(\exists P \in \Lambda_{t}(N)\right)\right\},
$$

where we set

$$
\omega_{\epsilon}:=d t-\sum_{i=1}^{N} t^{\epsilon_{i}} \theta_{i} d \theta_{i} \in \Omega^{1} .
$$

Note that for $\epsilon=\left(\epsilon_{i}\right)_{i=1}^{N}$ and $\epsilon^{\prime}=\left(\epsilon_{i}^{\prime}\right)_{i=1}^{N}$, if $\epsilon_{i} \equiv \epsilon_{i}^{\prime} \quad(\bmod 2)$ for all $i$, then $K(N ; \epsilon) \simeq K\left(N ; \epsilon^{\prime}\right)$ as Lie superalgebra. In the sequel, we assume that $\epsilon_{i} \in\{0,1\}$ for all $i$. For simplicity, we set

$$
\epsilon^{N S}:=(0, \ldots, 0), \quad \epsilon^{R}:=(1, \ldots, 1) .
$$

Remark that $K\left(N ; \epsilon^{N S}\right)$ and $K\left(N ; \epsilon^{R}\right)$ are respectively called the NeveuSchwarz sector and the Ramond sector.

To define Z-gradation of these Lie superalgebras, we introduce some notation. For $f \in \Lambda_{t}(N)$, we set

$$
\begin{aligned}
& \Delta(f):=2 f-\sum_{i=1}^{N} \theta_{i} \frac{\partial f}{\partial \theta_{i}}, \\
& D^{\epsilon}:=\frac{d}{d t}-\frac{1}{2} t^{-1} \sum_{i=1}^{N} \epsilon_{i} \theta_{i} \frac{\partial}{\partial \theta_{i}}, \\
& D_{f}:=(\Delta f) D^{\epsilon}+D^{\epsilon}(f) \sum_{i=1}^{N} \theta_{i} \frac{\partial}{\partial \theta_{i}}+(-1)^{|f|} \sum_{i=1}^{N} t^{-\epsilon_{i}} \frac{\partial f}{\partial \theta_{i}} \frac{\partial}{\partial \theta_{i}} .
\end{aligned}
$$

Notice that, the map

$$
\Lambda_{t}(N) \longrightarrow K(N ; \epsilon) \quad\left(f \mapsto D_{f}\right)
$$


is an isomorphism of vector spaces. Furthermore, for $f, g \in \Lambda_{t}(N)$, we have $\left[D_{f}, D_{g}\right]=D_{\{f, g\}}$, where we set

$$
\{f, g\}:=(\Delta f) D^{\epsilon}(g)-D^{\epsilon}(f)(\Delta g)+(-1)^{|f|} \sum_{i=1}^{N} t^{-\epsilon_{i}} \frac{\partial f}{\partial \theta_{i}} \frac{\partial g}{\partial \theta_{i}} .
$$

We introduce Z-gradation of $K(N ; \epsilon)$ as follows: Set $L_{n}:=-\frac{1}{2} D_{t^{n+1}}$ for $n \in \mathbf{Z}$. Then, $\left[L_{m}, L_{n}\right]=(m-n) L_{m+n}$ by Lemma 5.1, and

$$
K(N ; \epsilon)=\bigoplus_{m \in \mathbb{Z}} K(N ; \epsilon)^{m}, \quad K(N ; \epsilon)^{m}:=\left\{x \in K(N ; \epsilon) \mid\left[L_{0}, x\right]=-\frac{m}{2} x\right\} .
$$

Moreover, each graded subspace are described as follows:

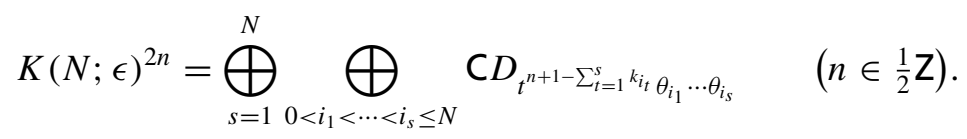

Let $K^{\prime}(N, \epsilon)$ be the derived subalgebra of $K(N, \epsilon)$. Then,

$$
K(N, \epsilon)=\left\{\begin{array}{ll}
K^{\prime}(N, \epsilon) & N \neq 4 \vee \sum_{i} \epsilon_{i} \notin 2 Z \\
K^{\prime}(N, \epsilon) \oplus C D_{t^{1-\sum_{i}} k_{i} \theta_{1} \theta_{2} \cdots \theta_{N}} & N=4 \wedge \sum_{i} \epsilon_{i} \in 2 Z
\end{array},\right.
$$

where $k_{i}:=\frac{1-\epsilon_{i}}{2}$ for each $i=1,2, \ldots, N$. We introduce Z-gradation of $K^{\prime}(N ; \epsilon)$ by $K^{\prime}(N ; \epsilon)^{m}:=K^{\prime}(N ; \epsilon) \cap K(N ; \epsilon)^{m}(m \in \mathbf{Z}) . K^{\prime}(N, \epsilon)$ is a simple Z-graded subalgebra of $W(N)$ with the Z-gradation (20) defined by $\beta_{i}=k_{i}$ for any $i$.

Remark 5.2. Non-trivial central extensions of $K^{\prime}(N ; \epsilon)$ for $N=0,1,2,3$ and 4 are respectively the so called Virasoro algebra, the $N=1,2,3$ and the big $N=4$ superconformal algebras.

5.1.4. Lie superalgebra $\mathrm{CK}_{6}(\epsilon)$. After the above examples of superconformal algebras were presented in [9], a new superconformal algebra $C K_{6}$ (the Neveu-Schwarz sector) was discovered in [3] and independently in [7]. Here, we introduce not only the Neveu-Schwarz sector but also other sectors of $\mathrm{CK}_{6}$.

It is convenient to use the following notation. For an ordered set $\left\{i_{1}, \ldots, i_{s}\right\}$ such that $i_{1}, \ldots, i_{s} \in\{1,2, \ldots, N\}$, we set

$$
\theta_{i_{1} \cdots i_{s}}:=\theta_{i_{1}} \cdots \theta_{i_{s}} \in K(N ; \epsilon) .
$$


For each $\left\{i_{1}, \ldots, i_{s}\right\}$, there exists an ordered set $\left\{j_{1}, \ldots, j_{N-s}\right\}$ which satisfy

$$
\theta_{i_{1} \cdots i_{s}} \theta_{j_{1} \cdots j_{N-s}}=\theta_{12 \cdots N}
$$

We denote the element $\theta_{j_{1} \cdots j_{N-s}} \in K(N ; \epsilon)$ by $\theta_{i_{1} \cdots i_{s}}^{*}$. For simplicity, we set $\theta_{\emptyset}^{*}:=\theta_{12 \cdots N}$. We also set

$$
k_{i_{1} \cdots i_{s}}:=\sum_{t=1}^{s} k_{i_{t}}, \quad k_{i_{1} \cdots i_{s}}^{*}:=k_{12 \cdots N}-k_{i_{1} \cdots i_{s}},
$$

and $k_{\emptyset}^{*}:=k_{12 \cdots N}$, where $k_{i}:=\frac{1-\epsilon_{i}}{2}$.

Suppose that $\epsilon=\left(\epsilon_{1}, \ldots, \epsilon_{6}\right)$ satisfies

$$
\sum_{i=1}^{6} \epsilon_{i} \equiv 0 \quad(\bmod 2) .
$$

For each $\beta \in\{ \pm \sqrt{-1}\}$, we define $\Phi_{m}, \Phi_{m}^{p q}, \Phi_{m}^{p}, \Phi_{m}^{p q r} \in K(6 ; \epsilon)^{2 m}$ as follows:

$$
\begin{aligned}
& \Phi_{m}:=-\frac{1}{2} t^{m+1}-\frac{\beta}{2}\left(m^{3}-m\right) t^{m+2-k_{\emptyset}^{*}} \theta_{\emptyset} \quad(m \in \mathrm{Z}), \\
& \Phi_{m}^{p q}:=t^{m+1-k_{p q}} \theta_{p q}-\beta m t^{m+1-k_{p q}^{*}} \theta_{p q}^{*} \quad\left(m \in k_{p q}+\mathrm{Z}\right), \\
& \Phi_{m}^{p}:=t^{m+1-k_{p}} \theta_{p}-\beta\left(m^{2}-\frac{1}{4}\right) t^{m+1-k_{p}^{*} \theta_{i}^{*}} \quad\left(m \in k_{p}+\mathrm{Z}\right), \\
& \Phi_{m}^{p q r}:=t^{m+1-k_{p q r}} \theta_{p q r}+\beta t^{m+1-k_{p q r}^{*}} \theta_{p q r}^{*} \quad\left(m \in k_{p q r}+\mathrm{Z}\right),
\end{aligned}
$$

where we identify $f \in \Lambda_{t}(6)$ with $D_{f} \in K(6 ; \epsilon)$ via the isomorphism (22).

We denote the Z-graded subspace of $K(6 ; \epsilon)$ spanned by the above vectors by $C K_{6}(\epsilon)$. By Proposition 5.4 and 5.5 in Subsection 5.4, $C K_{6}(\epsilon)$ is a simple Z-graded subalgebra of $K(6 ; \epsilon)$.

Here, we call $C K_{6}\left(\epsilon^{N S}\right)$ (resp. $\left.C K_{6}\left(\epsilon^{R}\right)\right)$ the Neveu-Schwarz sector (resp. the Ramond sector). Note that the Ramond sector $C K_{6}\left(\epsilon^{R}\right)$ has appeared in [10].

5.1.5. Lie superalgebra $C K_{4}(\epsilon)$. The small $N=4$ superconformal algebras have sectors other than the Neveu-Schwarz and the Ramond sectors, although, they are not considered in [9]. To treat all sectors of the small $N=4$ superconformal algebras in a unified manner, we introduce a simple Z-graded subalgebra $C K_{4}(\epsilon)$ of $K^{\prime}(4 ; \epsilon)$ in a way similar to Subsection 5.1.4. 
Throughout Section 5.1.5, we suppose that $\epsilon=\left(\epsilon_{1}, \ldots, \epsilon_{4}\right)$ satisfies

$$
\sum_{i=1}^{4} \epsilon_{i} \equiv 0 \quad(\bmod 2) .
$$

We use notation similar to Subsection 5.1.4. For each $\beta \in\{ \pm 1\}$, define $\Psi_{m}$, $\Psi_{m}^{p q}, \Psi_{m}^{p} \in K(4 ; \epsilon)^{2 m}$ as follows:

$$
\begin{aligned}
& \Psi_{m}:=-\frac{1}{2} t^{m+1}-\frac{\beta}{2}\left(m^{2}+m\right) t^{m+1-k_{\emptyset}^{*}} \theta_{\emptyset}^{*} \quad(m \in \mathbf{Z}), \\
& \Psi_{m}^{p q}:=t^{m+1-k_{p q}} \theta_{p q}-\beta t^{m+1-k_{p q}^{*}} \theta_{p q}^{*} \quad\left(m \in k_{p q}+\mathrm{Z}\right), \\
& \Psi_{m}^{p}:=t^{m+1-k_{p}} \theta_{p}-\beta\left(m+\frac{1}{2}\right) t^{m+1-k_{p}^{*}} \theta_{p}^{*} \quad\left(m \in k_{p}+\mathrm{Z}\right),
\end{aligned}
$$

where we identify $f \in \Lambda_{t}(4)$ with $D_{f} \in K(4 ; \epsilon)$ via the isomorphism (22).

We denote the Z-graded subspace of $K(4 ; \epsilon)$ spanned by the above vectors by $C K_{4}(\epsilon)$. By definition, we have $C K_{4}(\epsilon) \subset K^{\prime}(4 ; \epsilon)$. Moreover, Proposition 5.1 and 5.3 in Subsection 5.4 imply that $C K_{4}(\epsilon)$ is a simple Z-graded subalgebra of $K^{\prime}(4 ; \epsilon)$.

By Proposition 5.2, we have

$$
S^{\prime}(2 ; 0) \simeq C K_{4}\left(\epsilon^{N S}\right), \quad S^{\prime}(2 ;-1) \simeq C K_{4}\left(\epsilon^{R}\right)
$$

as Z-graded Lie superalgebra, and hence, we call $C K_{4}\left(\epsilon^{N S}\right)$ (resp. $\left.C K_{4}\left(\epsilon^{R}\right)\right)$ the Neveu-Schwarz sector (resp. the Ramond sector). Remark that the isomorphism $S^{\prime}(2 ; 0) \simeq C K_{4}\left(\epsilon^{N S}\right)$ was mentioned in [3].

Remark 5.3. As far as the authors know, $C K_{4}(\epsilon)$ and $C K_{6}(\epsilon)\left(\epsilon \neq \epsilon^{N S}, \epsilon^{R}\right)$, which are simple Z-graded Lie superalgebras, have not appeared in the literature.

5.1.6. On classification of physical superconformal algebras. First, we recall the definition of physical superconformal algebras [8]. Throughout Section 5.1.6, let $g$ be a Lie superalgebra, and let $a(z)=\sum_{n \in Z} a_{n} z^{-n-1}$ and $b(z)=\sum_{n \in Z} b_{n} z^{-n-1}$ be formal distributions with coefficients in $\mathfrak{g}$, i.e, $a_{n}, b_{n} \in \mathfrak{g}$ for any $n \in \mathbf{Z}$.

We say that $a(z)$ and $b(z)$ are local if $(z-w)^{k}[a(z), b(w)]=0$ for some $k \in \mathbf{Z}_{>0}$. If $a(z)$ and $b(z)$ are local, then there exist $c^{j}(z)(0 \leq j \leq k-1)$ such that

$$
[a(z), b(w)]=\sum_{j=0}^{k-1} c^{j}(w) \frac{1}{j !} \partial_{w}^{j} \delta(z-w),
$$


where $\delta(z-w):=z^{-1} \sum_{n \in Z}\left(\frac{z}{w}\right)^{n} \cdot c^{j}(z)$ is often denoted by $a(z)_{(j)} b(z)$ and is called the $j$ th product of $a(z)$ and $b(z)$. A formal distribution $L(z)$ is called the Virasoro distribution if it satisfies

$$
[L(z), L(w)]=\partial_{w} L(w) \delta(z-w)+2 L(w) \partial_{w} \delta(z-w),
$$

which is equivalent to $\left[L_{m}, L_{n}\right]=(m-n) L_{m+n}$, where $L(z)=\sum_{n \in Z} L_{n} z^{-n-2}$. Moreover, we call $a(z)$ a primary distribution of conformal weight $\Delta$ with respect to $L(z)$ if it satisfies

$$
[L(z), a(w)]=\partial_{w} a(w) \delta(z-w)+\Delta a(w) \partial_{w} \delta(z-w) .
$$

In the sequel, let $F$ be a set of formal distributions which satisfies

(1) $g$ is spanned by the coefficients of the formal distributions in $F$,

(2) any two formal distributions in $F$ are local.

$(\mathfrak{g}, F)$ is called a formal distribution Lie superalgebra.

Definition 5.1. A formal distribution Lie superalgebra $(\mathfrak{g}, F)$ is called a physical superconformal algebra if it satisfies

$\mathrm{C} 1$ the cardinality of $F$ is finite and $\mathrm{C}\left[\partial_{z}\right] F$ is closed under the $j$ th product for any $j \in \mathbf{Z}_{\geq 0}$.

$\mathrm{C} 2 \mathrm{~g}$ has no non-trivial ideal which is spanned by the coefficients of formal distributions in a $\mathrm{C}\left[\partial_{z}\right]$-submodule of $\mathrm{C}\left[\partial_{z}\right] F$.

C3 $F$ contains a Virasoro distribution $L(z)$, and any even (resp. odd) distribution in $F$ is $L(z)$ or a primary distribution of conformal weight 1 (resp. a primary distribution of conformal weight $\frac{1}{2}$ or $\frac{3}{2}$ ).

Remark that $L_{0}$ of the Virasoro distribution $L(z)$ defines a Z-gradation of a physical superconformal algebra.

The classification of the physical superconformal algebras can be described as follows:

THeOREm 5.1 ([8], cf. [15]). A physical superconformal algebra is isomorphic to one of $K^{\prime}\left(N ; \epsilon^{N S}\right)$ with $N=0,1,2,3,4, S^{\prime}(2 ; 0)\left(\simeq C K_{4}\left(\epsilon^{N S}\right)\right)$, $C K_{6}\left(\epsilon^{N S}\right)$ and $W(2)$ with Z-gradation (20) defined by $\left(\beta_{1}, \beta_{2}\right)=\left(\frac{1}{2}, \frac{1}{2}\right)$.

Although only the Neveu-Schwarz sectors appear in the theorem, the Ramond and the other sectors also play prominent roles both in mathematics and physics. In the following section, we determine the semi-infinite characters of all of them, namely, we deal with the next simple Z-graded Lie superalgebras: 


\begin{tabular}{|l|l|}
\hline$K^{\prime}(N ; \epsilon)$ & $N=0,1,2,3,4$ and $\epsilon_{i} \in\{0,1\}(1 \leq i \leq N)$ \\
\hline$C K_{4}(\epsilon)$ & $\epsilon_{i} \in\{0,1\}(1 \leq i \leq 4)$ s.t. $\sum_{i=1}^{4} \epsilon_{i} \equiv 0 \quad(\bmod 2)$ \\
\hline$C K_{6}(\epsilon)$ & $\epsilon_{i} \in\{0,1\}(1 \leq i \leq 6)$ s.t. $\sum_{i=1}^{6} \epsilon_{i} \equiv 0 \quad(\bmod 2)$ \\
\hline$W(2)$ & $\beta_{i} \in\left\{\frac{1}{2}, 0\right\}(1 \leq i \leq 2)$ \\
\hline
\end{tabular}

We also remark that a wider class of formal distribution Lie superalgebras (without the condition C3) are classified in [6], however, the definition of a formal distribution Lie superalgebra does not allow a Z-gradation of the Lie superalgebra a priori, because of the absence of the Virasoro distribution.

\subsection{Semi-infinite characters}

Some simple Z-graded Lie superalgebras listed above do not have the semiinfinite characters, and we need to consider their central extensions. Before computing semi-infinite characters, we briefly recall the relation between a central extension of a Lie superalgebra and its two cocycle.

For a two cocycle $\psi$ of a Lie superalgebra $\mathfrak{a}$, the central extension of $\mathfrak{a}$ defined by $\psi$ is the Lie superalgebra $\mathfrak{g}:=\mathfrak{a} \oplus \mathrm{C} c$ with the bracket

$$
\left[x_{1}+r_{1} c, x_{2}+r_{2} c\right]_{\mathfrak{g}}:=\left[x_{1}, x_{2}\right]+\psi\left(x_{1}, x_{2}\right) c,
$$

where [, ] is the Lie bracket of $a$. We denote $[,]_{\mathfrak{g}}$ by $[$, ] for simplicity.

5.2.1. $K^{\prime}(N ; \epsilon)$ with $N=0,1,2,3,4$. We first recall some facts on central extensions of $K^{\prime}(N ; \epsilon)$. For a complete description of the central extensions of $K^{\prime}(N ; \epsilon)$, see [9].

Let $\psi$ be a two cocycle of $K^{\prime}(N ; \epsilon)$ and $\mathfrak{g}=K^{\prime}(N ; \epsilon) \oplus \mathrm{C} c$ the central extension of $K^{\prime}(N ; \epsilon)$ defined by $\psi$. Remark that in cases where $N=3,4$, by Lemma 2.4 and Theorem 5.2, for any two cocycle $\psi$ of $K^{\prime}(N ; \epsilon)$ and any semi-infinite character $\gamma$ of $\mathfrak{g}$, we have

$$
\gamma(c)=0 .
$$

Hence, we need an explicit form of a two cocycle $\psi$ only for $N=0,1,2$. The following $\psi$ gives a non-trivial central extension of $K^{\prime}(N ; \epsilon)(N=0,1,2)$.

Case $N=0$ (the Virasoro algebra):

$$
\psi\left(D_{t^{m+1}}, D_{t^{n+1}}\right)=\frac{1}{3} \delta_{m+n, 0}\left(m^{3}-m\right) .
$$


Case $N=1$ :

$$
\begin{aligned}
& \psi\left(D_{t^{m+1}}, D_{t^{n+1}}\right)=\frac{1}{3} \delta_{m+n, 0}\left(m^{3}-m\right), \\
& \psi\left(D_{t^{m+1-k_{1}} \theta_{1}}, D_{t^{n+1-k_{1}} \theta_{1}}\right)=\frac{1}{3} \delta_{m+n, 0}\left(m^{2}-\frac{1}{4}\right),
\end{aligned}
$$

and the other pairs are zero.

Case $N=2$ :

$$
\begin{aligned}
& \psi\left(D_{t^{m+1}}, D_{t^{n+1}}\right)=\frac{1}{3} \delta_{m+n, 0}\left(m^{3}-m\right), \\
& \psi\left(D_{t^{m+1-k_{i}} \theta_{i}}, D_{t^{n+1-k_{j}} \theta_{j}}\right)=\frac{1}{3} \delta_{i, j} \delta_{m+n, 0}\left(m^{2}-\frac{1}{4}\right), \\
& \psi\left(D_{t^{m+1-k_{1}-k_{2}} \theta_{1} \theta_{2}}, D_{t^{n+1-k_{1}-k_{2}} \theta_{1} \theta_{2}}\right)=-\frac{1}{3} \delta_{m+n, 0} m,
\end{aligned}
$$

and the other pairs are zero.

In the sequel, let $\psi$ be the above cocycle of $K^{\prime}(N ; \epsilon)$ for $N=0,1,2$ and an arbitrary two cocycle for $N=3,4$. We regard $g:=K^{\prime}(N ; \epsilon) \oplus \mathcal{C} c$ as a Z-graded Lie superalgebra by setting

$$
\mathrm{g}^{m}:=\left\{\begin{array}{ll}
K^{\prime}(N ; \epsilon)^{m} \oplus \mathrm{C}_{c} & m=0 \\
K^{\prime}(N ; \epsilon)^{m} & m \neq 0
\end{array} .\right.
$$

The following is one of the main results of this section.

THEOREM 5.2. The semi-infinite character $\gamma \in \operatorname{Hom}_{\mathrm{C}}\left(\mathfrak{h}_{\overline{0}}, \mathrm{C}_{\overline{0}}^{0}\right)$ of $\mathrm{g}$ is given as follows:

Case $N=0$ :

$$
\gamma(c)=26, \quad \gamma\left(D_{t}\right)=-2 \text {. }
$$

Case $N=1$ :

$$
\gamma(c)=15, \quad \gamma\left(D_{t}\right)=-\frac{5}{4}+\frac{1}{4} \delta_{\epsilon_{1}, 0} .
$$

Case $N=2$ :

$$
\begin{aligned}
& \gamma(c)=6, \\
& \gamma\left(D_{t}\right)=-\frac{1}{2}+\frac{1}{2} \delta_{\epsilon_{1}, 0} \delta_{\epsilon_{2}, 0}, \\
& \gamma\left(D_{t^{1-k_{1}-k_{2}} \theta_{1} \theta_{2}}\right)=0 \quad\left(\text { if } k_{1}+k_{2} \in \mathrm{Z}\right) .
\end{aligned}
$$


Case $N=3$ :

$$
\begin{aligned}
& \gamma(c)=0, \\
& \gamma\left(D_{t}\right)=\prod_{i=1}^{3} \delta_{\epsilon_{i}, 0}, \\
& \gamma\left(D_{t^{1-k_{i}-k_{j}} \theta_{i} \theta_{j}}\right)=0 \quad\left(\text { if } k_{i}+k_{j} \in \mathbf{Z}\right) .
\end{aligned}
$$

Case $N=4$ :

$$
\begin{aligned}
& \gamma(c)=0, \\
& \gamma\left(D_{t}\right)=2 \prod_{i=1}^{4} \delta_{\epsilon_{i}, 0}, \\
& \gamma\left(D_{t^{1-k_{i}-k_{j}} \theta_{i} \theta_{j}}\right)=0 \quad\left(\text { if } k_{i}+k_{j} \in \mathbf{Z}\right) .
\end{aligned}
$$

Proof. Here, we show the theorem in the two simplest cases, i.e., $N=0$ and $N=1$. The other cases can be proved similarly.

We first consider the $N=0$ (the Virasoro algebra) case. Let $\omega$ be the critical cocycle of $\mathrm{g}$. Notice that $\mathrm{g}=\bigoplus_{m \in 2 Z} \mathrm{~g}^{m}$, where

$$
\mathfrak{h}=\mathrm{C} D_{t} \oplus \mathrm{C} c, \quad \mathrm{~g}^{m}=\mathrm{C} D_{t^{1+\frac{m}{2}}} \quad(m \neq 0) .
$$

Since $\mathfrak{g}$ is generated by $\mathfrak{g}^{-4} \oplus \mathfrak{g}^{-2} \oplus \mathfrak{h} \oplus \mathfrak{g}^{2} \oplus \mathfrak{g}^{4}$, Lemma 2.3 implies that $\gamma \in \operatorname{Hom}_{C}\left(\mathfrak{h}_{\overline{0}}, \mathrm{C}_{\overline{0}}^{0}\right)$ is the semi-infinite character of $g$ if and only if

$$
\omega(x, y)=-\gamma([x, y]) \quad\left(\forall x, y \in \mathfrak{g}^{-4} \oplus \mathfrak{g}^{-2} \oplus \mathfrak{h} \oplus \mathfrak{g}^{2} \oplus \mathfrak{g}^{4}\right) .
$$

Using Lemma 2.1, by direct computation, we have

$$
\omega\left(D_{t}, D_{t}\right)=0, \quad \omega\left(D_{t^{2}}, D_{1}\right)=-8, \quad \omega\left(D_{t^{3}}, D_{t^{-1}}\right)=-68 .
$$

Since $\left[D_{t}, D_{t}\right]=0,\left[D_{t^{2}}, D_{1}\right]=-4 D_{t}$ and $\left[D_{t^{3}}, D_{t^{-1}}\right]=-8 D_{t}+2 c,(31)$ holds for $\gamma$ given in this theorem. Hence, the theorem holds in the $N=0$ case.

Next, we consider the $N=1$ case. Remark that the Z-gradation of each sector is given by

The Neveu-Schwarz sector $\left(\epsilon_{1}=0\right)$ :

$$
\mathfrak{h}=\mathrm{C} D_{t} \oplus \mathrm{C} c, \quad \mathrm{~g}^{m}= \begin{cases}\mathrm{C} D_{t^{\frac{m}{2}+1}} & (m \in 2 \mathrm{Z}) \\ \mathrm{C} D_{t^{\frac{m+1}{2}} \theta_{1}} & (m \in 2 \mathrm{Z}+1)\end{cases}
$$


The Ramond sector $\left(\epsilon_{1}=1\right)$ :

$\mathfrak{h}=\mathrm{CD}_{t} \oplus \mathrm{C} D_{t \theta_{1}} \oplus \mathrm{C} c, \quad \mathrm{~g}^{m}=\left\{\begin{array}{ll}\mathrm{C} D_{t^{\frac{m}{2}+1}} \oplus \mathrm{C} D_{t^{\frac{m}{2}+1} \theta_{1}} & (m \in 2 \mathrm{Z}) \\ \{0\} & (m \in 2 \mathrm{Z}+1)\end{array}\right.$,

and the Neveu-Schwarz (resp. the Ramond) sector is generated by $\bigoplus_{|m| \leq 3} \mathrm{~g}^{m}$ (resp. $\bigoplus_{|m| \leq 2} \mathrm{~g}^{m}$ ). Moreover, by direct computation, we have

The Neveu-Schwarz sector

$\omega\left(D_{t \theta_{1}}, D_{\theta_{1}}\right)=-1, \quad \omega\left(D_{t^{2}}, D_{1}\right)=-4, \quad \omega\left(D_{t^{2} \theta_{1}}, D_{t^{-1} \theta_{1}}\right)=-11$,

The Ramond sector

$$
\omega\left(D_{t^{2}}, D_{1}\right)=-5, \quad \omega\left(D_{t^{2} \theta_{1}}, D_{\theta_{1}}\right)=-5 .
$$

Hence, an argument similar to the previous case yields the theorem for this case.

5.2.2. $C K_{4}(\epsilon)$. Let $\mathfrak{g}=C K_{4}(\epsilon) \oplus \mathrm{C} c$ be the central extension of $C K_{4}(\epsilon)$ defined by the following two cocycle $\psi$ :

$$
\begin{aligned}
\psi\left(\Psi_{m}, \Psi_{n}\right) & :=\frac{1}{12}\left(m^{3}-m\right) \delta_{m+n, 0}, \\
\psi\left(\Psi_{m}^{i j}, \Psi_{n}^{p q}\right) & :=\frac{1}{3} m \delta_{m+n, 0} \delta_{i, p} \delta_{j, q} \quad(i<j, p<q) \\
\psi\left(\Psi_{m}^{i}, \Psi_{n}^{j}\right) & :=-\frac{1}{3}\left(m^{2}-\frac{1}{4}\right) \delta_{m+n, 0} \delta_{i, j} .
\end{aligned}
$$

We introduce Z-gradation of $\mathfrak{g}$ in a way similar to (30).

THEOREM 5.3. The semi-infinite character $\gamma \in \operatorname{Hom}_{\mathrm{C}}\left(\mathfrak{h}_{\overline{0}}, \mathrm{C}_{\overline{0}}^{0}\right)$ of $\mathrm{g}$ is given as follows:

(1) Case $\epsilon=(0,0,0,0)$ :

$$
\begin{aligned}
& \gamma(c)=-12, \\
& \gamma\left(\Psi_{0}\right)=-1, \\
& \gamma\left(\Psi_{0}^{i j}\right)=0 \quad((i, j)=(1,2),(2,3),(3,1)) .
\end{aligned}
$$


(2) Case $\epsilon=(1,1,1,1)$ :

$$
\begin{aligned}
& \gamma(c)=-12, \\
& \gamma\left(\Psi_{0}\right)=-\frac{1}{2}, \\
& \gamma\left(\Psi_{0}^{i j}\right)=0 \quad((i, j)=(1,2),(2,3),(3,1)) .
\end{aligned}
$$

(3) Case $\epsilon=(1,1,0,0)$ :

$$
\begin{aligned}
& \gamma(c)=-12, \\
& \gamma\left(\Psi_{0}\right)=-\frac{1}{2}, \\
& \gamma\left(\Psi_{0}^{i j}\right)=0 \quad((i, j)=(1,2)) .
\end{aligned}
$$

By using the commutation relations in Proposition 5.1, one can directly show this theorem in a way similar to Theorem 5.2.

ReMARK 5.4. The central charge $\gamma(c)$ of the semi-infinite character $\gamma$ of the simple Z-graded Lie superalgebras in Subsection 5.2.1 and 5.2.2 is called the critical dimension in the superstring theory (e.g., [9]). Although, the value $\gamma(c)$ may not necessarily coincide with the critical dimension in some references because of the normalization of the central element $c$, this value coincides with the central charge in the condition that the square of the BRST charge vanishes (see Subsection 2.4).

\begin{tabular}{|l|c|c|c|c|c|c|}
\hline & $N=0$ & $N=1$ & $N=2$ & $N=3$ & (big) $N=4$ & (small) $N=4$ \\
\hline$\gamma(c)$ & 26 & 15 & 6 & 0 & 0 & -12 \\
\hline
\end{tabular}

5.2.3. $C K_{6}(\epsilon)$. The Z-graded Lie superalgebra $C K_{6}(\epsilon)$ has the semi-infinite character without central extension. Indeed, by using the commutation relations in Proposition 5.4, one can check the following theorem by direct computation.

THEOREM 5.4. The semi-infinite character $\gamma \in \operatorname{Hom}_{\mathcal{C}}\left(\mathfrak{h}_{\overline{0}}, \mathrm{C}_{\overline{0}}^{0}\right)$ of $\mathrm{CK}_{6}(\epsilon)$ is given as follows:

(1) Case $\epsilon=(0,0,0,0,0,0)$ :

$$
\gamma\left(\Phi_{0}\right)=-2, \quad \gamma\left(\Phi_{0}^{i j}\right)=0 \quad(1 \leq i<j \leq 6) .
$$


(2) Case $\epsilon=(1,1,1,1,1,1)$ :

$$
\gamma\left(\Phi_{0}\right)=0, \quad \gamma\left(\Phi_{0}^{i j}\right)=0 \quad(1 \leq i<j \leq 6) .
$$

(3) Case $\epsilon=(1,1,0,0,0,0)$ :

$$
\gamma\left(\Phi_{0}\right)=0, \quad \gamma\left(\Phi_{0}^{i j}\right)=0 \quad((i, j)=(1,2) \text { or } 3 \leq i<j \leq 6) .
$$

(4) Case $\epsilon=(1,1,1,1,0,0)$ :

$$
\gamma\left(\Phi_{0}\right)=0, \quad \gamma\left(\Phi_{0}^{i j}\right)=0 \quad(1 \leq i<j \leq 4 \text { or }(i, j)=(5,6)) .
$$

5.2.4. $W(2)$. Let $\mathrm{g}:=W(2) \oplus \mathrm{C} c$ be the non-trivial central extension defined by the following two cocycle $\psi$ :

$$
\begin{aligned}
& \psi\left(t^{k} \theta_{1} \frac{\partial}{\partial \theta_{2}}, t^{l} \theta_{2} \frac{\partial}{\partial \theta_{1}}\right)=-k \delta_{k+l, 0}, \quad \psi\left(t^{k} \theta_{1} \frac{\partial}{\partial \theta_{1}}, t^{l} \theta_{2} \frac{\partial}{\partial \theta_{2}}\right)=k \delta_{k+l, 0}, \\
& \psi\left(t^{k} \theta_{1} \theta_{2} \frac{\partial}{\partial \theta_{2}}, t^{l} \frac{\partial}{\partial \theta_{1}}\right)=k \delta_{k+l, 0}, \quad \psi\left(t^{k} \theta_{2} \theta_{1} \frac{\partial}{\partial \theta_{1}}, t^{l} \frac{\partial}{\partial \theta_{2}}\right)=k \delta_{k+l, 0} .
\end{aligned}
$$

We regard $g$ as a Z-graded Lie superalgebra in a way similar to (30). By direct computation, we have

THEOREM 5.5. The semi-infinite character $\gamma \in \operatorname{Hom}_{\mathcal{C}}\left(\mathfrak{h}_{\overline{0}}, \mathrm{C}_{\overline{0}}^{0}\right)$ of $\mathfrak{g}$ is given as follows:

(1) Case $\left(\beta_{1}, \beta_{2}\right)=\left(\frac{1}{2}, \frac{1}{2}\right)$ :

$$
\begin{aligned}
& \gamma(c)=1, \\
& \gamma\left(t \frac{\partial}{\partial t}\right)=1, \\
& \gamma\left(t \theta_{1} \theta_{2} \frac{\partial}{\partial t}\right)=\gamma\left(\theta_{i} \frac{\partial}{\partial \theta_{j}}\right)=0 \quad(\forall i, j \in\{1,2\}) .
\end{aligned}
$$

(2) Case $\left(\beta_{1}, \beta_{2}\right)=(0,0)$ :

$$
\begin{aligned}
& \gamma(c)=1, \\
& \gamma\left(t \frac{\partial}{\partial t}\right)=0, \\
& \gamma\left(t \theta_{1} \theta_{2} \frac{\partial}{\partial t}\right)=\gamma\left(\theta_{i} \frac{\partial}{\partial \theta_{j}}\right)=0 \quad(\forall i, j \in\{1,2\}) .
\end{aligned}
$$


(3) Case $\left(\beta_{1}, \beta_{2}\right)=\left(\frac{1}{2}, 0\right)$ :

$$
\gamma(c)=1, \quad \gamma\left(t \frac{\partial}{\partial t}\right)=0, \quad \gamma\left(\theta_{i} \frac{\partial}{\partial \theta_{i}}\right)=\left\{\begin{array}{ll}
0 & \text { if } i=1 \\
1 & \text { if } i=2
\end{array} .\right.
$$

\subsection{Contragredient Lie superalgebra}

In order to describe the Feigin-Arkhipov-Soergel duality for basic classical Lie superalgebras or some affine Lie superalgebras, here, we give the semi-infinite character of contragredient Lie superalgebras.

5.3.1. Definition. We recall the definition of a contragredient Lie superalgebra. Let $I$ be a finite index set, and let $\tau$ be a subset of $I$. Let $A=\left(a_{i, j}\right)_{i, j \in I}$ be a complex matrix of rank $l$. Let $\mathfrak{h}$ be a $(2 n-l)$-dimensional $\mathrm{C}$-vector space. Let $\Pi:=\left\{\alpha_{i} \mid i \in I\right\}$ (resp. $\left\{h_{i} \mid i \in I\right\}$ ) be a linearly independent finite subset of $\mathfrak{h}^{*}\left(\right.$ resp. h) ), which satisfies $\alpha_{i}\left(h_{j}\right)=a_{j, i}$ for any $i, j \in I$. We set $Q:=\bigoplus_{i \in I} Z \alpha_{i}$.

Definition 5.2. A Lie superalgebra $g$ over $C$ is called a contragredient Lie superalgebra if

(1) $\mathfrak{g}$ is $Q$-graded, i.e., $\mathfrak{g}=\bigoplus_{\alpha \in Q} \mathfrak{g}_{\alpha}$ and $\left[\mathfrak{g}_{\alpha}, \mathfrak{g}_{\beta}\right] \subset \mathfrak{g}_{\alpha+\beta}$ for any $\alpha, \beta \in Q$,

(2) there exist $e_{i}$ and $f_{i}(i \in I)$ such that

(a) $\mathfrak{g}$ is generated by $\left\{e_{i}, f_{i}, h \mid i \in I, h \in \mathfrak{h}\right\}$,

(b) $\mathfrak{g}_{0}=\mathfrak{h}, \mathfrak{g}_{\alpha_{i}}=\mathrm{C} e_{i}$ and $\mathfrak{g}_{-\alpha_{i}}=\mathrm{C} f_{i}$,

(c) for any $h, h^{\prime} \in \mathfrak{h}$ and $i, j \in I$,

$$
\begin{array}{ll}
|h|=\overline{0}, & \left|e_{i}\right|=\left|f_{i}\right|=\left\{\begin{array}{ll}
\overline{0} & i \notin \tau \\
\overline{1} & i \in \tau
\end{array},\right. \\
{\left[h, h^{\prime}\right]=0,} & {\left[e_{i}, f_{j}\right]=\delta_{i, j} h_{i},} \\
{\left[h, e_{i}\right]=\alpha_{i}(h) e_{i},} & {\left[h, f_{i}\right]=-\alpha_{i}(h) f_{i},}
\end{array}
$$

(3) every $Q$-graded ideal $\mathfrak{i}$ of $\mathfrak{g}$ such that $\mathfrak{i} \cap \mathfrak{h}=\{0\}$ is zero.

For $\alpha \in Q$ such that $\alpha=\sum_{i \in I} m_{i} \alpha_{i}$, set ht $\alpha:=\sum_{i \in I} m_{i}$. For $n \in \mathbf{Z}$, set $\mathrm{g}^{n}:=\bigoplus_{\text {ht } \alpha=n} \mathfrak{g}_{\alpha}$. Then, $\mathfrak{g}=\bigoplus_{n \in Z} \mathfrak{g}^{n}$ is a Z-graded Lie superalgebra with

$$
\mathfrak{g}^{1}=\bigoplus_{i \in I} \mathrm{C} e_{i}, \quad \mathfrak{g}^{0}=\mathfrak{h}, \quad \mathfrak{g}^{-1}=\bigoplus_{i \in I} \mathrm{C} f_{i}
$$

5.3.2. Semi-infinite characters of contragredient Lie superalgebras. Let $\rho$ be an element of $\mathfrak{h}^{*}$, which satisfies

$$
\rho\left(h_{i}\right)=\frac{1}{2} \alpha_{i}\left(h_{i}\right) \quad(\forall i \in I) .
$$


One can show the following theorem by direct computation.

Theorem 5.6. The semi-infinite character $\gamma \in \operatorname{Hom}_{\mathrm{C}}\left(\mathfrak{h}, \mathrm{C}_{\overline{0}}^{0}\right)$ of a contragredient Lie superalgebra $\mathfrak{g}$ is given by

$$
\gamma=-2 \rho .
$$

\subsection{Appendix: Simplicity of $C K_{4}(\epsilon)$ and $C K_{6}(\epsilon)$}

In this subsection, we prove that $C K_{4}(\epsilon)$ is a simple Z-graded subalgebra of $K^{\prime}(4 ; \epsilon)$. Here, we omit the proof for $C K_{6}(\epsilon)$, since a similar argument works.

5.4.1. $C K_{4}(\epsilon)$. Recall that for each $\epsilon=\left(\epsilon_{1}, \ldots, \epsilon_{4}\right)$ such that $\sum_{i=1}^{4} \epsilon_{i} \equiv 0$ $(\bmod 2)$ and $\beta \in\{ \pm 1\}, C K_{4}(\epsilon)$ is the Z-graded subspace spanned by the vectors (28). By direct computation, we have

Proposition 5.1. The following commutation relations hold.

$$
\begin{aligned}
& \left\{\Psi_{m}, \Psi_{n}\right\}=(m-n) \Psi_{m+n}, \\
& \left\{\Psi_{m}, \Psi_{n}^{i j}\right\}=-n \Psi_{m+n}^{i j}, \\
& \left\{\Psi_{m}, \Psi_{n}^{i}\right\}=\left(\frac{m}{2}-n\right) \Psi_{m+n}^{i}, \\
& \left\{\Psi_{m}^{i j}, \Psi_{n}^{p q}\right\}=\left\{\begin{array}{ll}
0 & \text { if } \sharp(\{i, j\} \cap\{p, q\})=0,2 \\
2 \delta_{i, p} \Psi_{m+n}^{j q}-2 \delta_{i, q} \Psi_{m+n}^{j p} & \text { if } \sharp(\{i, j\} \cap\{p, q\})=1 \\
-2 \delta_{j, p} \Psi_{m+n}^{i q}+2 \delta_{j, q} \Psi_{m+n}^{i p}
\end{array},\right. \\
& \left\{\Psi_{m}^{i j}, \Psi_{n}^{p}\right\}=\left\{\begin{array}{ll}
\delta_{i, p} \Psi_{m+n}^{j}-\delta_{j, p} \Psi_{m+n}^{i} & \text { if } p \in\{i, j\} \\
-\sum_{q} \beta \epsilon^{i j p q} \Psi_{m+n}^{q} & \text { if } p \notin\{i, j\}
\end{array},\right. \\
& \left\{\Psi_{m}^{i}, \Psi_{n}^{p}\right\}=\left\{\begin{array}{ll}
2 \Psi_{m+n} & \text { if } i=p \\
-(m-n) \Psi_{m+n}^{i p} & \text { if } i \neq p
\end{array},\right.
\end{aligned}
$$

where

$$
\epsilon^{i j p q}:=\left\{\begin{array}{ll}
\operatorname{sgn}\left(\begin{array}{l}
1234 \\
i j p q
\end{array}\right) & \text { if }\{i, j, p, q\} \text { are distinct } \\
0 & \text { otherwise }
\end{array} .\right.
$$

Hence, $\mathrm{CK}_{4}(\epsilon)$ is a Z-graded subalgebra of $K^{\prime}(4 ; \epsilon)$. 
The Neveu-Schwarz and the Ramond sectors of $C K_{4}(\epsilon)$ are respectively isomorphic to $S^{\prime}(2 ; 0)$ and $S^{\prime}(2 ;-1)$. To describe the isomorphisms, we recall the following fact. For each $n \in \mathrm{Z}$, let $L_{n}$ be the vector defined in (21) and

$$
\tilde{L}_{n}:=-t^{n+1} \frac{\partial}{\partial t}-(n+\alpha+1) t^{n}\left(\theta_{1} \frac{\partial}{\partial \theta_{1}}+\theta_{2} \frac{\partial}{\partial \theta_{2}}\right) .
$$

Notice that $\theta_{i} \tilde{L}_{n} \in S^{\prime}(2 ; \alpha)$ for $i=1,2$. Then, each graded subspace $S^{\prime}(2 ; \alpha)^{m}$ $(m \in Z)$ can be described as follows:

(1) The Neveu-Schwarz sector $(n \in Z)$ :

$$
\begin{aligned}
S^{\prime}(2 ; \alpha)^{2 n} & =\mathrm{C} L_{n} \oplus \mathrm{C} t^{n}\left(\theta_{1} \frac{\partial}{\partial \theta_{1}}-\theta_{2} \frac{\partial}{\partial \theta_{2}}\right) \oplus \mathrm{C} t^{n} \theta_{1} \frac{\partial}{\partial \theta_{2}} \oplus \mathrm{C} t^{n} \theta_{2} \frac{\partial}{\partial \theta_{1}}, \\
S^{\prime}(2 ; 0)^{2 n+1} & =\mathrm{C} t^{n+1} \frac{\partial}{\partial \theta_{1}} \oplus \mathrm{C} t^{n+1} \frac{\partial}{\partial \theta_{2}} \oplus \mathrm{C} \theta_{1} \tilde{L}_{n} \oplus \mathrm{C} \theta_{2} \tilde{L}_{n} .
\end{aligned}
$$

(2) The Ramond sector $(n \in Z)$ :

$$
\begin{aligned}
S^{\prime}(2 ;-1)^{2 n}= & \mathrm{C} L_{n} \oplus \mathrm{C} t^{n}\left(\theta_{1} \frac{\partial}{\partial \theta_{1}}-\theta_{2} \frac{\partial}{\partial \theta_{2}}\right) \oplus \mathrm{C} t^{n} \theta_{1} \frac{\partial}{\partial \theta_{2}} \\
& \oplus \mathrm{C} t^{n} \theta_{2} \frac{\partial}{\partial \theta_{1}} \oplus \mathrm{C} t^{n} \frac{\partial}{\partial \theta_{1}} \oplus \mathrm{C} t^{n} \frac{\partial}{\partial \theta_{2}} \oplus \mathrm{C} \theta_{1} \tilde{L}_{n} \oplus \mathrm{C} \theta_{2} \tilde{L}_{n}, \\
S^{\prime}(2 ;-1)^{2 n+1}= & \{0\} .
\end{aligned}
$$

We set

$$
\epsilon_{\alpha}:=\left\{\begin{array}{ll}
\epsilon^{N S} & \alpha=0 \\
\epsilon^{R} & \alpha=-1
\end{array}, \quad k_{\alpha}:=\left\{\begin{array}{ll}
\frac{1}{2} & \alpha=0 \\
0 & \alpha=-1
\end{array} .\right.\right.
$$

Proposition 5.2. For $\alpha \in\{0,-1\}$, the following map $C_{4}\left(\epsilon_{\alpha}\right) \rightarrow S^{\prime}(2 ; \alpha)$ gives an isomorphism of Z-graded Lie superalgebras.

$$
\begin{array}{lll}
\Psi_{m} \longmapsto L_{m}, & \Psi_{m}^{1} \longmapsto\left(t^{m+k_{\alpha}} \frac{\partial}{\partial \theta_{2}}+\theta_{2} \tilde{L}_{m-k_{\alpha}}\right) \\
\Psi_{m}^{12} \longmapsto-\sqrt{-1} t^{m}\left(\theta_{1} \frac{\partial}{\partial \theta_{1}}-\theta_{2} \frac{\partial}{\partial \theta_{2}}\right), & \Psi_{m}^{2} \longmapsto-\sqrt{-1}\left(t^{m+k_{\alpha}} \frac{\partial}{\partial \theta_{2}}-\theta_{2} \tilde{L}_{m-k_{\alpha}}\right) \\
\Psi_{m}^{23} \longmapsto-\sqrt{-1} t^{m}\left(\theta_{1} \frac{\partial}{\partial \theta_{2}}+\theta_{2} \frac{\partial}{\partial \theta_{1}}\right), & \Psi_{m}^{3} \longmapsto\left(t^{m+k_{\alpha}} \frac{\partial}{\partial \theta_{1}}+\theta_{1} \tilde{L}_{m-k_{\alpha}}\right) \\
\Psi_{m}^{31} \longmapsto t^{m}\left(-\theta_{1} \frac{\partial}{\partial \theta_{2}}+\theta_{2} \frac{\partial}{\partial \theta_{1}}\right), & \Psi_{m}^{4} \longmapsto-\sqrt{-1} \beta\left(t^{m+k_{\alpha}} \frac{\partial}{\partial \theta_{1}}-\theta_{1} \tilde{L}_{m-k_{\alpha}}\right)
\end{array}
$$

Finally, we show the simplicity (as Z-graded Lie superalgebra) of $C K_{4}(\epsilon)$. 
Proposition 5.3. For each $\epsilon=\left(\epsilon_{1}, \ldots, \epsilon_{4}\right)$ such that $\sum_{i=1}^{4} \epsilon_{i} \equiv 0$ $(\bmod 2), C_{4}(\epsilon)$ is a simple Z-graded Lie superalgebra, i.e., it has no nontrivial Z-graded ideal.

Proof. Since $S^{\prime}(2 ; \alpha)$ are simple Z-graded Lie superalgebras, the simplicity for the Neveu-Schwarz and the Ramond sectors follows from the above isomorphisms. Here, we only consider the other sectors.

We may assume that $\epsilon=(1,1,0,0)$. Set $g:=C K_{4}(\epsilon)$. One can directly show that

LEMMA 5.2.

(1) For any $m \in \mathbf{Z}_{\geq 0}$, if $x \in \mathfrak{g}^{m}$ commutes with $\mathfrak{g}^{-1}$, i.e., $\left\{\mathfrak{g}^{-1}, x\right\}=\{0\}$, then $x=0$.

(2) For any $m \in \mathbf{Z}_{<-1}$, if $x \in \mathfrak{g}^{m}$ commutes with $\mathrm{g}^{1}$, i.e., $\left\{\mathfrak{g}^{1}, x\right\}=\{0\}$, then $x=0$.

(3) We set

$$
\mathfrak{a}^{ \pm}:=\mathrm{C}\left(\Psi_{-\frac{1}{2}}^{23} \pm \sqrt{-1} \Psi_{-\frac{1}{2}}^{31}\right) \oplus \mathrm{C}\left(\Psi_{-\frac{1}{2}}^{3} \mp \sqrt{-1} \beta \Psi_{-\frac{1}{2}}^{4}\right) .
$$

Then, $\mathfrak{a}^{ \pm}$are irreducible $\mathfrak{h}$-submodules of $\mathfrak{g}^{-1}$ which satisfy $\mathfrak{g}^{-1}=\mathfrak{a}^{+} \oplus$ $\mathfrak{a}^{-}$and $\mathfrak{a}^{+} \nsucceq \mathfrak{a}^{-}$as $\mathfrak{h}$-module.

The first and the second statements imply that any non-zero Z-graded ideal $\mathfrak{i}=\bigoplus_{n \in \mathcal{Z}} \dot{\mathfrak{i}}^{n}\left(\mathfrak{i}^{n}:=\mathfrak{i} \cap \mathfrak{g}^{n}\right)$ of $\mathfrak{g}$ satisfies

$$
i^{-1} \neq\{0\} .
$$

Hence, by the third statement we have

$$
\mathfrak{a}^{+} \subset \mathfrak{i} \quad \text { or } \quad \mathfrak{a}^{-} \subset \mathfrak{i} .
$$

This implies that $\Psi_{0} \in \mathfrak{i}$ since

$$
\left\{\Psi_{-\frac{1}{2}}^{3}+\sqrt{-1} \beta \Psi_{-\frac{1}{2}}^{4}, \Psi_{-\frac{1}{2}}^{3}-\sqrt{-1} \beta \Psi_{-\frac{1}{2}}^{4}\right\}=4 \Psi_{-1}, \quad\left\{\Psi_{1}, \Psi_{-1}\right\}=2 \Psi_{0} .
$$

Using this fact, we obtain $\mathrm{g}^{m} \subset \mathfrak{i}$ for $m \neq 0$, and thus, $\mathfrak{i}=\mathfrak{g}$.

5.4.2. $C K_{6}(\epsilon)$. Recall that for each $\epsilon=\left(\epsilon_{1}, \ldots, \epsilon_{6}\right)$ such that $\sum_{i=1}^{6} \epsilon_{i} \equiv 0$ $(\bmod 2)$ and $\beta \in\{ \pm \sqrt{-1}\}, C K_{6}(\epsilon)$ is defined as the Z-graded subspace of $K(6 ; \epsilon)$ spanned by the vectors $(26)$. 
Proposition 5.4. The following commutation relations hold:

$\left\{\Phi_{m}, \Phi_{n}\right\}=(m-n) \Phi_{m+n}$,

$\left\{\Phi_{m}, \Phi_{n}^{i j}\right\}=-n \Phi_{m+n}^{i j}$,

$\left\{\Phi_{m}, \Phi_{n}^{i}\right\}=\left(\frac{m}{2}-n\right) \Phi_{m+n}^{i}$,

$\left\{\Phi_{m}, \Phi_{n}^{i j k}\right\}=-\left(\frac{m}{2}+n\right) \Phi_{m+n}^{i j k}$,

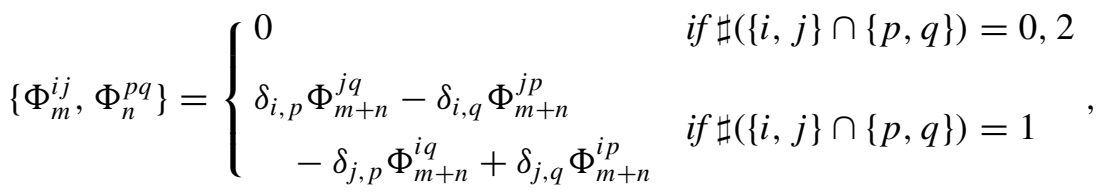

$\left\{\Phi_{m}^{i j}, \Phi_{n}^{p}\right\}=\left\{\begin{array}{ll}\delta_{i, p} \Phi_{m+n}^{j}-\delta_{j, p} \Phi_{m+n}^{i} & \text { if } p \in\{i, j\} \\ -m \Phi_{m+n}^{i j p} & \text { if } p \notin\{i, j\}\end{array}\right.$,

$\left\{\Phi_{m}^{i j}, \Phi_{n}^{p q r}\right\}=\left\{\begin{array}{ll}0 & \text { if } \sharp(\{i, j\} \cap\{p, q, r\})=0,2 \\ \delta_{i, p} \Phi_{m+n}^{j q r}-\delta_{i, q} \Phi_{m+n}^{j p r} & \\ +\delta_{i, r} \Phi_{m+n}^{j p q}-\delta_{j, p} \Phi_{m+n}^{i q r} & \text { if } \sharp(\{i, j\} \cap\{p, q, r\})=1 \\ & +\delta_{j, q} \Phi_{m+n}^{i p r}-\delta_{j, r} \Phi_{m+n}^{i p q}\end{array}\right.$, ,

$\left\{\Phi_{m}^{i}, \Phi_{n}^{p}\right\}= \begin{cases}2 \Phi_{m+n} & \text { if } i=p \\ -(m-n) \Phi_{m+n}^{i p} & \text { if } i \neq p\end{cases}$

$\left\{\Phi_{m}^{i}, \Phi_{n}^{p q r}\right\}=\left\{\begin{array}{ll}-\delta_{i, p} \Phi_{m+n}^{q r}+\delta_{i, q} \Phi_{m+n}^{p r}-\delta_{i, r} \Phi_{m+n}^{p q} & \text { if } i \in\{p, q, r\} \\ \sum_{k<l} \beta \epsilon^{k l i p q r} \Phi_{m+n}^{k l} & \text { if } i \notin\{p, q, r\}\end{array}\right.$,

$\left\{\Phi_{m}^{i j k}, \Phi_{n}^{p q r}\right\}=0$

$\Phi_{m}^{p q r}=\beta \epsilon^{p q r i j k} \Phi_{m}^{i j k}$, if $\{p, q, r\} \cap\{i, j, k\}=\emptyset$,

where

$$
\epsilon^{\text {klipqr }}:=\left\{\begin{array}{ll}
\operatorname{sgn}\left(\begin{array}{l}
123456 \\
\text { klipqr }
\end{array}\right) & \text { if }\{k, l, i, p, q, r\} \text { are distinct } \\
0 & \text { otherwise }
\end{array} .\right.
$$

Hence, $C_{6}(\epsilon)$ is a Z-graded subalgebra of $K(6 ; \epsilon)$. 
Similarly to Proposition 5.3, one can show that

Proposition 5.5. For each $\epsilon=\left(\epsilon_{1}, \ldots, \epsilon_{6}\right)$ such that $\sum_{i=1}^{6} \epsilon_{i} \equiv 0$ $(\bmod 2), K_{6}(\epsilon)$ is a simple Z-graded Lie superalgebra.

\section{REFERENCES}

1. Arkhipov, S., Semi-infinite cohomology of associative algebras and bar duality, Internat. Math. Res. Notices 17 (1997), 833-863.

2. Brundan, J., Tilting modules for Lie superalgebras, Comm. Algebra 32 (2004), 2251-2268.

3. Cheng, S-J., and Kac, V. G., A new $N=6$ superconformal algebra, Comm. Math. Phys. 186 (1997), 219-231.

4. Eguchi, T., and Taormina, A., Character formulas for $N=4$ superconformal algebra, Phys. Lett. B 200 (1988), 315-322.

5. Feigin, B. L., Semi-infinite homology of Lie, Kac-Moody and Virasoro algebras, Uspekhi Mat. Nauk 39 (1984), 195-196.

6. Fattori, D., and Kac, V. G., Classification of finite simple Lie conformal superalgebras, J. Alg. 258 (2002), 23-59.

7. Grozman, P., Leites D. A., and Shchepochina, I. M., Lie superalgebras of string theories, Acta Math. Vietnam. 26, No. 1 (2001), 27-63.

8. Kac, V. G., Superconformal algebras and transitive group actions on quadrics, Comm. Math. Phys. 186 (1997), 233-252. Erratum: Comm. Math. Phys. 217 (2001), 697-698.

9. Kac, V. G., and van de Leur, J. W., On the classification of superconformal algebras, String '88, World Scientific (1989), 77-106.

10. Kac, V. G., Martinez, C., and Zelmanov, E., Graded simple Jordan superalgebras of growth one, Mem. Amer. Math. Soc. 150 (2001), 711.

11. Lian, B. H., and Zuckerman, G. J., BRST cohomology and highest weight vectors I, Comm. Math. Phys. 135 (1991), 547-580.

12. Soergel, W., Character formulas for tilting modules over Kac-Moody algebras, Represent. Theory 2 (1998), 432-448.

13. Schwimmer, A., and Seiberg, N., Comments on the $N=2,3,4$ superconformal algebras in two dimensions, Phys. Lett. B 184 (1987), 191-196.

14. Voronov, A., Semi-infinite homological algebra, Invent. Math. 113 (1993), 103-146.

15. Yamamoto, G., Algebraic structures on quasi-primary states in superconformal algebras, J. Math. Soc. Japan 57 (2005), 309-332.

DEPARTMENT OF MATHEMATICS

FACULTY OF SCIENCE

KOBE UNIVERSITY

KOBE 657-8501

JAPAN

E-mail: iohara@math.kobe-u.ac.jp
DEPARTMENT OF MATHEMATICS

FACULTY OF ENGINEERING

UNIVERSITY OF FUKUI

FUKUI 910-8507

JAPAN

E-mail: koga@quantum.apphy.fukui-u.ac.jp 